\title{
Total Synthesis of (+)-Lithospermic Acid by Asymmetric Intramolecular Alkylation via Catalytic C-H Bond Activation
}

Steven J. O’Malley, Kian L. Tan, Anja F. Watzke, Robert G. Bergman* and Jonathan A. Ellman*

Department of Chemistry, University of California-Berkeley, Berkeley, CA 94720

\section{Supporting Information}

\section{Experimental:}

General Methods. Unless otherwise noted, materials were obtained from commercial suppliers and used without further purification. THF was distilled under $\mathrm{N}_{2}$ from sodium benzophenone ketyl, pyridine from calcium hydride, and methanol from magnesium methoxide immediately prior to use. Degassed toluene was purified by passage through an activated alumina column. Methyl chloroformate was purchased from Acros, quinoline was purchased from Aldrich, and (R)-(-)-aminoindane was purchased from Lancaster, and all were distilled prior to use. All organic reactions were performed under an atmosphere of $\mathrm{N}_{2}$ in flame-dried or oven-dried glassware unless otherwise stated. All C-H activation experiments were prepared in a $\mathrm{N}_{2}$-filled Vac inert atmosphere box. The following compounds were prepared according to referenced literature procedures: $\left[\mathrm{RhCl}(\mathrm{coe})_{2}\right]_{2}{ }^{1}$ and $\mathrm{FCPCy} 2 .{ }^{2}$ Thin-layer chromatography was performed on Merck $60 \mathrm{~F}_{254} 250-\mu \mathrm{m}$ silica gel plates. Visualization of the developed chromatogram was performed by fluorescence quenching, $\mathrm{KMnO}_{4}$ stain, or paraanisaldehyde stain. Flash chromatography was carried out according to the general procedure of Still ${ }^{3}$ using Merck 60 230-240 mesh silica gel or activity I basic $\mathrm{Al}_{2} \mathrm{O}_{3}$ as noted. Organic extracts were dried over $\mathrm{MgSO}_{4}$ or $\mathrm{Na}_{2} \mathrm{SO}_{4}$ and were concentrated using

(1) Van der Ent, A.; Onderdelinden, A.L. Inorg. Synth. 1973, 14, 92-93.

(2) Guillaneaux, D.; Kagan, H. J. Am. Chem. Soc. 1995, 60, 2502.

(3) Still, W.C.; Kahn, M.; Mitra, A. J. Org. Chem. 1978, 43, 2923-2925. 
a Buchi rotary evaporator under high vacuum pressure. Melting points were determined on a Mel-Temp apparatus and are uncorrected. IR spectra were recorded on a Nicolet Avatar 360 FTIR spectrometer equipped with an attenuated total reflectance accessory, and only partial data are listed. Unless otherwise noted, ${ }^{1} \mathrm{H}$ and ${ }^{13} \mathrm{C}$ NMR spectra were obtained in $\mathrm{CDCl}_{3}$. NMR chemical shifts are reported in ppm and referenced to residual protonated solvent. Mass spectra were performed by the University of California, Berkeley Micro-Mass Facility. Chiral HPLC analyses were performed on an Agilent 1100 system with a Chiral PAK AS column $(250 \mathrm{~mm} 4.6 \mathrm{~mm})$ with a flow rate of 1 $\mathrm{mL} / \mathrm{min}$ and with $i-\mathrm{PrOH} / \mathrm{hexanes}$ as the mobile phase. Reverse phase preparative HPLC purifications were performed on an Agilent 1100 system using a MicrosorbTM $\mathrm{C}_{18}(250$ x $21.4 \mathrm{~mm}$ ) column with a flow rate of $20 \mathrm{~mL} / \mathrm{min}$ and with acetonitrile/water $0.1 \%$ TFA as the mobile phase. A Perkin-Elmer 241 polarimeter with a sodium lamp was used to determine specific rotations and concentrations are reported in $\mathrm{g} / \mathrm{dL}$. The sense of induction in the asymmetric alkylation by $\mathrm{C}-\mathrm{H}$ bond activation reaction was ascertained through derivatization to a crystalline hydrazone derivative. 


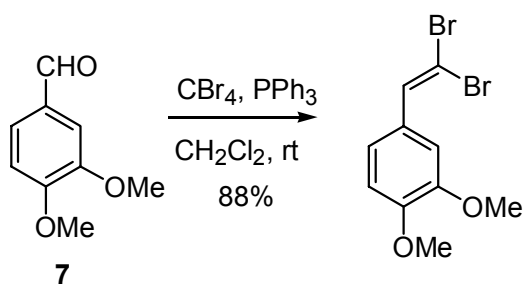

4-(2,2-Dibromo-vinyl)-1,2-dimethoxy-benzene. Carbon tetrabromide (34.67 g, 104.5 mmol, 1.0 equiv) and $\mathrm{CH}_{2} \mathrm{Cl}_{2}(175 \mathrm{~mL})$ were added to a $500 \mathrm{~mL}$ round bottom flask equipped with a magnetic stir bar and septum. The reaction flask was cooled to $0{ }^{\circ} \mathrm{C}$ with an ice bath for $10 \mathrm{~min}$. Triphenylphosphine (54.84 g, $209.1 \mathrm{mmol}, 2.0$ equiv) was added in portions over $5 \mathrm{~min}$, and the dark red solution was stirred at $0{ }^{\circ} \mathrm{C}$ for $30 \mathrm{~min} .3,4-$ Dimethoxybenzaldehyde $(17.37 \mathrm{~g}, 104.5 \mathrm{mmol}, 1.0$ equiv) was added and the mixture was stirred at $0{ }^{\circ} \mathrm{C}$ for $1 \mathrm{~h}$. A 1:1 mixture of $\mathrm{H}_{2} \mathrm{O}$ :brine was added and the layers were separated. The aqueous layer was extracted with a 1:1 mixture of hexanes: $\mathrm{CH}_{2} \mathrm{Cl}_{2}$, and the combined organic layers were dried, filtered, and concentrated under reduced pressure. The crude material was chromatographed on $\mathrm{SiO}_{2}(0-15 \%$ EtOAc/hexanes as eluent) to afford $29.37 \mathrm{~g}(88 \%)$ of the dibromide as a yellow oil. IR (ZnSe): 2994, 2955, 2928, 2897, 2831, 1592, 1507, 1456, 1433, 1417, 1254, 1223, 1146, $1017 \mathrm{~cm}^{-1} .{ }^{1} \mathrm{H}$ NMR $\left(400 \mathrm{MHz}, \mathrm{CDCl}_{3}\right): \delta 7.41\left(\mathrm{~s}, 1 \mathrm{H}, \mathbf{H C}=\mathrm{CBr}_{2}\right), 7.19(\mathrm{~s}, 1 \mathrm{H}, \operatorname{ArH}), 7.10(\mathrm{~d}, 1 \mathrm{H}, J=7.9 \mathrm{~Hz}$, $\operatorname{ArH}), 6.86(\mathrm{~d}, 1 \mathrm{H}, J=8.4 \mathrm{~Hz}, \operatorname{ArH}), 3.90\left(\mathrm{~s}, 3 \mathrm{H}, \mathrm{OCH}_{3}\right), 3.89$ (s, 3H, OCH 3$) .{ }^{13} \mathrm{C} \mathrm{NMR}$ $\left(100 \mathrm{MHz}, \mathrm{CDCl}_{3}\right): \delta 148.9,148.1,136.0,127.4,121.6,110.7,110.4,86.8,55.5,55.4$. EI(+)-HRMS Calcd. for $\mathrm{C}_{10} \mathrm{H}_{10} \mathrm{O}_{2} \mathrm{Br}^{81} \mathrm{Br}[\mathrm{M}]^{+}$: 321.9027 . Found: 321.9024 . 


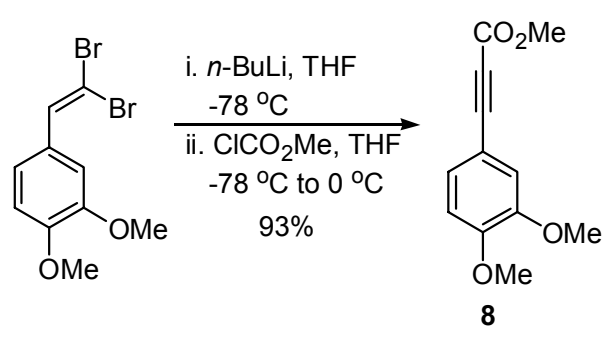

(3,4-Dimethoxy-phenyl)-propynoic acid methyl ester (8). Dibromide (4.41 g, 13.79

mmol, 1.0 equiv) and THF (45 mL) were added to a $250 \mathrm{~mL}$ round bottom flask equipped with a magnetic stir bar and septum, and the reaction flask was cooled to $-78{ }^{\circ} \mathrm{C} . n$-BuLi (11.6 $\mathrm{mL}, 2.5 \mathrm{M}$ in hexanes, 2.1 equiv) was added slowly via syringe and the reaction solution was stirred at $-78{ }^{\circ} \mathrm{C}$ for $45 \mathrm{~min}$ and then at $0{ }^{\circ} \mathrm{C}$ for $45 \mathrm{~min}$. The flask was recooled to $-78{ }^{\circ} \mathrm{C}$, and freshly distilled methyl chloroformate $(1.1 \mathrm{~mL}, 1.3 \mathrm{mmol}, 1.0$ equiv) was added slowly via syringe. The mixture was stirred at $-78{ }^{\circ} \mathrm{C}$ for $10 \mathrm{~min}$, then $0{ }^{\circ} \mathrm{C}$ for $1 \mathrm{~h}$. Saturated aqueous $\mathrm{NH}_{4} \mathrm{Cl}$ solution was added, and the layers were separated. The aqueous layer was extracted with $\mathrm{Et}_{2} \mathrm{O}$, and the combined organic layers were dried, filtered and concentrated under reduced pressure. The residue was chromatographed on $\mathrm{SiO}_{2}(2-20 \% \mathrm{EtOAc} /$ hexanes as eluent) to afford $2.83 \mathrm{~g}(93 \%)$ of ester 8 as a white solid. Melting point: $76-77^{\circ} \mathrm{C}$. IR (ZnSe): 3002, 2967, 2936, 2893, 2835, 2202, 1693, 1592 , 1503, 1437, 1247, 1223, 1134, $1010 \mathrm{~cm}^{-1} .{ }^{1} \mathrm{H}$ NMR (400 MHz, $\left.\mathrm{CDCl}_{3}\right): \delta 7.23(\mathrm{~d}, 1 \mathrm{H}, J$ $=8.3 \mathrm{~Hz}, \operatorname{ArH}), 7.07(\mathrm{~s}, 1 \mathrm{H}, \operatorname{ArH}), 6.85(\mathrm{~d}, 1 \mathrm{H}, J=8.3 \mathrm{~Hz}, \operatorname{ArH}), 3.91\left(\mathrm{~s}, 3 \mathrm{H}, \mathrm{OCH}_{3}\right)$, $3.88\left(\mathrm{~s}, 3 \mathrm{H}, \mathrm{OCH}_{3}\right), 3.84\left(\mathrm{~s}, 3 \mathrm{H}, \mathrm{OCH}_{3}\right) .{ }^{13} \mathrm{C} \mathrm{NMR}\left(75 \mathrm{MHz}, \mathrm{CDCl}_{3}\right): \delta 154.3,151.3$, 148.5, 127.0, 114.9, 111.0, 110.8, 87.1, 79.3, 55.7, 55.7, 52.4. EI(+)-HRMS Calcd. for $\mathrm{C}_{12} \mathrm{H}_{12} \mathrm{O}_{4}[\mathrm{M}]^{+}:$220.0736. Found 220.0736. 

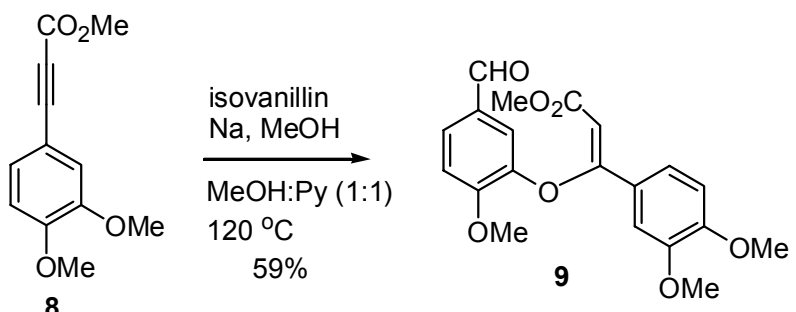

\section{3-(3,4-Dimethoxy-phenyl)-3-(5-formyl-2-methoxy-phenoxy)-acrylic acid methyl}

ester (9). To a sealed tube equipped with a magnetic stir bar was added $\mathrm{MeOH}(55 \mathrm{~mL})$ followed by $\mathrm{Na}$ metal $(2.10 \mathrm{~g}, 91.5 \mathrm{mmol}, 1.6$ equiv, cut into small pieces). Once all the $\mathrm{Na}$ had dissolved, isovanillin $(64.1 \mathrm{~g}, 421 \mathrm{mmol}, 7.4$ equiv) was added and the reaction mixture became a thick, yellow slurry. After stirring at room temperature for $5 \mathrm{~min}$, pyridine (55 mL) was added, followed by 8 (12.6 g, $57.2 \mathrm{mmol}, 1.0$ equiv), and the reaction mixture was stirred at $120{ }^{\circ} \mathrm{C}$ for $1 \mathrm{~h} 45 \mathrm{~min}$. The mixture was cooled to room temperature, diluted with EtOAc, and saturated aqueous $\mathrm{NH}_{4} \mathrm{Cl}$ solution was added. The layers were separated and the organic layer was washed several times with saturated aqueous $\mathrm{K}_{2} \mathrm{CO}_{3}$ solution to remove excess isovanillin. The combined organic layers were dried, filtered and concentrated under reduced pressure. The crude material was chromatographed on basic alumina $\left(0-20 \% \mathrm{EtOAc} / \mathrm{CH}_{2} \mathrm{Cl}_{2}\right.$ as eluent) to afford a mixture of $E$ and $Z$ 9, which was crystallized using $\mathrm{CH}_{2} \mathrm{Cl}_{2} /$ pentane mixtures to afford $4.99 \mathrm{~g}$ (23\%) of $Z$ 9. Purification of the mother liquor on $\mathrm{SiO}_{2}(5-75 \%$ EtOAc/hexanes as eluent) and subsequent crystallization with $\mathrm{CH}_{2} \mathrm{Cl}_{2}$ /pentane mixtures (repeated twice) afforded $6.94 \mathrm{~g}(33 \%)$ and $0.67 \mathrm{~g} \mathrm{(3 \% ),} \mathrm{respectively,} \mathrm{of} Z 9$ (12.6 g total, 59\% overall yield of pure $Z$ 9). Melting point: $144-145{ }^{\circ} \mathrm{C}$. IR (ZnSe): 3060, 3007, 2950, 2840, 1716, 1683, 1601, $1513,1448,1434,1344,1272,1257,1222,1190,1162,1127,1123,1018,915,878,809$ $\mathrm{cm}^{-1} .{ }^{1} \mathrm{H}$ NMR $\left(400 \mathrm{MHz}, \mathrm{CDCl}_{3}\right): \delta 9.72(\mathrm{~s}, 1 \mathrm{H}, \mathrm{CHO}), 7.50(\mathrm{dd}, 1 \mathrm{H}, J=1.8,8.3 \mathrm{~Hz}$, ArH), $7.27(\mathrm{~d}, 1 \mathrm{H}, J=1.8 \mathrm{~Hz}, \operatorname{ArH}), 7.21(\mathrm{dd}, 1 \mathrm{H}, J=2.0,8.5 \mathrm{~Hz}, \operatorname{ArH}), 7.10(\mathrm{~d}, 1 \mathrm{H}, J$ 
$=2.0 \mathrm{~Hz}, \operatorname{ArH}), 7.04(\mathrm{~d}, 1 \mathrm{H}, J=8.3 \mathrm{~Hz}, \operatorname{ArH}), 6.79(\mathrm{~d}, 1 \mathrm{H}, J=8.5 \mathrm{~Hz}, \operatorname{ArH}), 6.08(\mathrm{~s}, 1 \mathrm{H}$, $\left.\mathrm{CHCO}_{2} \mathrm{Me}\right), 4.04\left(\mathrm{~s}, 3 \mathrm{H}, \mathrm{OCH}_{3}\right), 3.85\left(\mathrm{~s}, 3 \mathrm{H}, \mathrm{OCH}_{3}\right), 3.82\left(\mathrm{~s}, 3 \mathrm{H}, \mathrm{OCH}_{3}\right), 3.66(\mathrm{~s}, 3 \mathrm{H}$, $\left.\mathrm{CO}_{2} \mathrm{CH}_{3}\right) .{ }^{13} \mathrm{C} \mathrm{NMR}\left(100 \mathrm{MHz}, \mathrm{CDCl}_{3}\right): \delta 190.2,164.4,162.2,154.3,151.2,148.8$, 146.6, 129.7, 127.5, 125.5, 120.5, 115.0, 111.4, 110.8, 109.1, 104.2, 56.1, 55.7, 55.6, 51.1. FAB(+)-HRMS Calcd. for $\mathrm{C}_{20} \mathrm{H}_{20} \mathrm{O}_{7}[\mathrm{M}]^{+}$: 372.1209. Found: 372.1213 . 

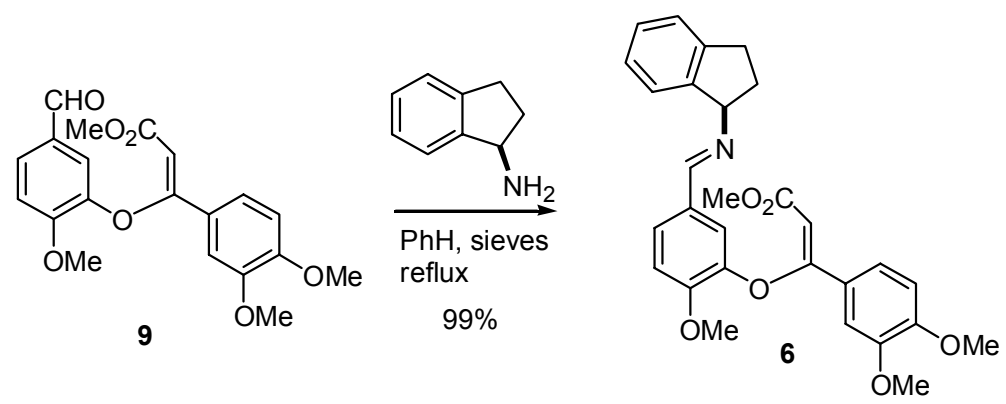

\section{3-(3,4-Dimethoxy-phenyl)-3-[5-(inan-1-yliminomethyl)-2-methoxy-phenoxy]-acrylic}

acid methyl ester (6). To a $50 \mathrm{~mL}$ round bottom flask equipped with a magnetic stir bar and septum was added 9 (1.02 g, $2.75 \mathrm{mmol}, 1.0$ equiv), benzene (14 mL), $4 \AA$ molecular sieves ( $(8.0 \mathrm{~g})$, and $(R)-(-)$-aminoindane $(0.385 \mathrm{~g}, 2.89 \mathrm{mmol}, 1.05$ equiv). The reaction flask was then equipped with a reflux condenser and the mixture was heated to reflux for $14 \mathrm{~h}$. The reaction mixture was cooled to room temperature, diluted with benzene, dried over $\mathrm{Na}_{2} \mathrm{SO}_{4}$, filtered through Celite ${ }^{\circledR}$ and concentrated. The residue was dissolved in minimal $\mathrm{CH}_{2} \mathrm{Cl}_{2}$ and precipitated with pentane to afford $1.33 \mathrm{~g}(99 \%)$ of 6 as a white foam. IR (ZnSe): 2946, 2361, 1719, 1636, 1601, 1579, 1510, 1457, 1431, 1347, 1264, $1218,1127,1064,1020,913,807,762,728 \mathrm{~cm}^{-1} .{ }^{1} \mathrm{H} \mathrm{NMR}\left(400 \mathrm{MHz}, \mathrm{CDCl}_{3}\right): \delta 8.18(\mathrm{~s}$, $1 \mathrm{H}, \mathrm{CH}=\mathrm{NCH}), 7.38(\mathrm{~d}, 1 \mathrm{H}, J=8.4 \mathrm{~Hz}, \operatorname{ArH}), 7.31-7.11(\mathrm{~m}, 6 \mathrm{H}, \operatorname{ArH} \times$ x $), 7.00(\mathrm{~d}, 1 \mathrm{H}$, $J=7.3 \mathrm{~Hz}, \operatorname{ArH}), 6.90(\mathrm{~d}, 1 \mathrm{H}, J=8.4 \mathrm{~Hz}, \operatorname{ArH}), 6.73(\mathrm{~d}, 1 \mathrm{H}, J=8.5 \mathrm{~Hz}, \operatorname{ArH}), 5.97(\mathrm{~s}$, $\left.1 \mathrm{H}, \mathrm{CHCO}_{2} \mathrm{CH}_{3}\right), 4.78\left(\mathrm{t}, 1 \mathrm{H}, J=7.2 \mathrm{~Hz}, \mathrm{CH}_{2} \mathrm{CHN}=\mathrm{CH}\right), 3.93\left(\mathrm{~s}, 3 \mathrm{H}, \mathrm{OCH}_{3}\right), 3.79(\mathrm{~s}$, $\left.3 \mathrm{H}, \mathrm{OCH}_{3}\right), 3.76\left(\mathrm{~s}, 3 \mathrm{H}, \mathrm{OCH}_{3}\right), 3.64\left(\mathrm{~s}, 3 \mathrm{H}, \mathrm{CO}_{2} \mathrm{CH}_{3}\right), 3.07-3.01(\mathrm{~m}, 1 \mathrm{H}$, one of $\left.\mathrm{ArCH}_{2} \mathrm{CH}_{2}\right), 2.92-2.84\left(\mathrm{~m}, 1 \mathrm{H}\right.$, one of $\left.\mathrm{ArCH}_{2} \mathrm{CH}_{2}\right), 2.39-2.35(\mathrm{~m}, 1 \mathrm{H}$, one of $\mathrm{ArCH}_{2} \mathrm{CH}_{2}$ ), 2.19-2.09 (m, 1H, one of $\left.\left.\mathrm{ArCH}_{2} \mathrm{CH}_{2}\right) .{ }^{13} \mathrm{C} \mathrm{NMR} \mathrm{(100} \mathrm{MHz,} \mathrm{CDCl}_{3}\right): \delta$ 164.6, 162.6, 159.0, 151.3, 150.8, 148.6, 146.0, 144.1, 143.4, 129.2, 128.0, 127.2, 126.0, 124.4, 124.0, 124.0, 120.5, 116.1, 111.6, 110.6, 109.5, 103.6, 74.5, 55.8, 55.5, 55.4, 50.9, 
33.9, 30.7. $[\alpha]_{D}\left(c 1.12, \mathrm{CH}_{2} \mathrm{Cl}_{2}\right)+7.7^{0}$. FAB(+)-HRMS Calcd. for $\mathrm{C}_{29} \mathrm{H}_{30} \mathrm{NO}_{6}[\mathrm{M}+\mathrm{H}]^{+}$: 488.2073. Found: 488.2079. 


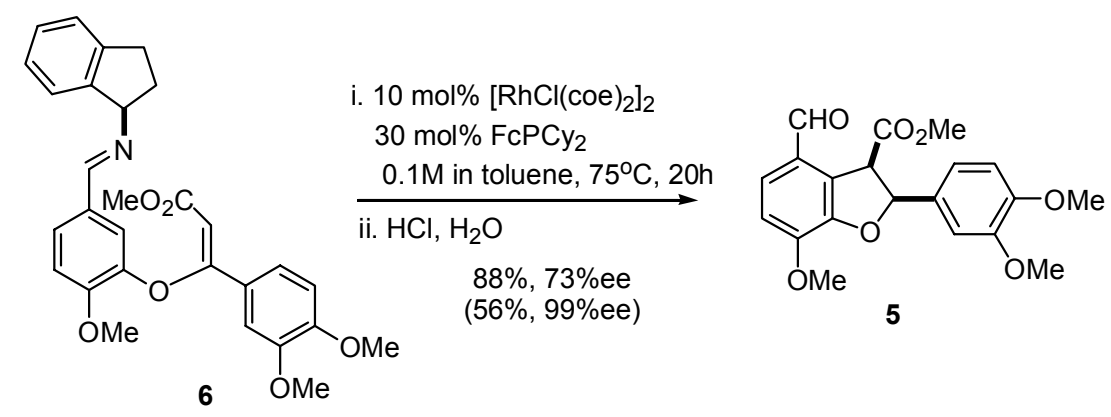

2-(3,4-Dimethoxy-phenyl)-4-formyl-7-methoxy-2,3-dihydro-benzofuran-3-carboxylic

acid methyl ester (5). In a dry box, a solution of $\left[\mathrm{RhCl}(\mathrm{coe})_{2}\right]_{2}(0.463 \mathrm{~g}, 0.644 \mathrm{mmol}$, 0.1 equiv) and $\mathrm{FcPCy}_{2}(0.739 \mathrm{mg}, 1.93 \mathrm{mmol}, 0.3$ equiv) in toluene (20 mL) was added to a sealed tube equipped with a magnetic stir bar. A solution of imine 6 ( $3.14 \mathrm{~g}, 6.44$ mmol, 1.0 equiv) in toluene (45 $\mathrm{mL})$ was then added and the reaction vessel was sealed and the solution was stirred at $75{ }^{\circ} \mathrm{C}$ for $20 \mathrm{~h}$. The reaction solution was cooled to room temperature, transferred to a round bottom flask with EtOAc rinses and concentrated under reduced pressure. The residue was washed with $1 \mathrm{~N} \mathrm{HCl} \mathrm{(aq)} \mathrm{and} \mathrm{stirred} \mathrm{for} 20$ min. The mixture was diluted with EtOAc, the layers were separated, and the aqueous layer was extracted with EtOAc. The combined organic layers were dried, filtered and concentrated under reduced pressure. The crude material was chromatographed on $\mathrm{SiO}_{2}$ (2-20\% EtOAc/hexanes as eluent) to afford $2.1 \mathrm{~g}(88 \%)$ of 5, which was recrystallized three times from benzene/pentane mixtures to afford $1.34 \mathrm{~g}(56 \%)$ of enantiopure 5 (determined by HPLC analysis) as a white solid. Melting point: $119-120{ }^{\circ} \mathrm{C} . \mathrm{IR}(\mathrm{ZnSe})$ : $3085,2983,2938,2893,2827,1728,1683,1614,1581,1512,1434,1283,1238,1140$, 1091, 1025, 943, 862, $805 \mathrm{~cm}^{-1} .{ }^{1} \mathrm{H}$ NMR (400 MHz, $\left.\mathrm{CDCl}_{3}\right): \delta 9.82$ (s, 1H, CHO), 7.39 $(\mathrm{d}, 1 \mathrm{H}, J=8.3 \mathrm{~Hz}, \operatorname{ArH}), 6.98(\mathrm{~d}, 1 \mathrm{H}, J=8.3 \mathrm{~Hz}, \operatorname{ArH}), 6.96-6.62(\mathrm{~m}, 2 \mathrm{H}, \operatorname{ArH}$ x 2), $6.82(\mathrm{~d}, 1 \mathrm{H}, J=8.2, \operatorname{ArH}), 6.00(\mathrm{~d}, 1 \mathrm{H}, J=10.2 \mathrm{~Hz}, \operatorname{ArOCHAr}), 4.89(\mathrm{~d}, 1 \mathrm{H}, J=10.2$ $\left.\mathrm{Hz}, \mathrm{ArCHCO}_{2} \mathrm{CH}_{3}\right), 3.97\left(\mathrm{~s}, 3 \mathrm{H}, \mathrm{OCH}_{3}\right), 3.85\left(\mathrm{~s}, 3 \mathrm{H}, \mathrm{OCH}_{3}\right), 3.84$ (s, 3H, OCH$\left.)_{3}\right), 3.23$ (s, 
$\left.3 \mathrm{H}, \mathrm{CO}_{2} \mathrm{CH}_{3}\right) .{ }^{13} \mathrm{C} \mathrm{NMR}\left(100 \mathrm{MHz}, \mathrm{CDCl}_{3}\right): \delta 190.6,169.7,149.9,149.5,149.0,148.5$, $128.7,128.2,126.4,125.3,119.4,111.5,110.3,109.4,87.7,56.0,55.8,55.7,54.1,51.7$. $[\alpha]_{D}\left(c\right.$ 0.95, $\left.\mathrm{CH}_{2} \mathrm{Cl}_{2}\right)-112.9^{\circ}$. FAB(+)-HRMS Calcd. for $\mathrm{C}_{20} \mathrm{H}_{20} \mathrm{O}_{7}[\mathrm{M}]^{+}: 372.1209$.

Found: 372.1213. HPLC analysis (AS column, 70\% hexanes: 30\% $i$-PrOH, 20 min wait time): peak $1=17.5 \mathrm{~min}$ (minor enantiomer) and peak $2=37.0 \mathrm{~min}$ (major enantiomer). 

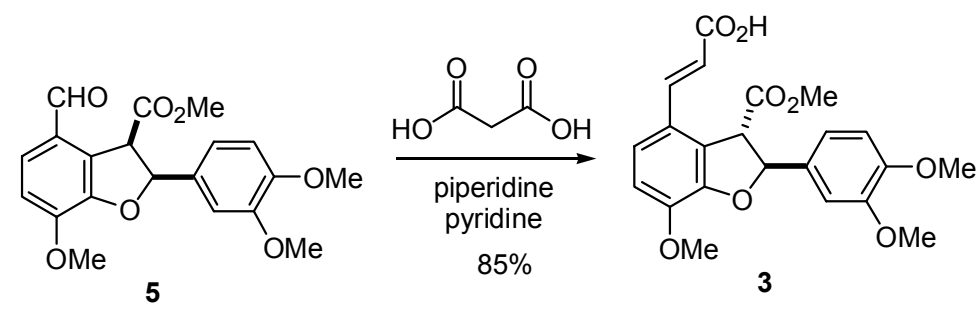

\section{4-(2-Carboxy-vinyl)-2-(3,4-dimethoxy-phenyl)-7-methoxy-2,3-dihydro-benzofuran-}

3-carboxylic acid methyl ester (3). To a round bottom flask equipped with a magnetic stir bar and septum was added 5 (0.259 g, 0.696 mmol, 1.0 equiv $)$, malonic acid ( $0.217 \mathrm{~g}$, $2.09 \mathrm{mmol}, 3.0$ equiv), pyridine $(23 \mathrm{~mL})$ and piperidine $(0.7 \mathrm{~mL})$. The reaction mixture was heated to $100{ }^{\circ} \mathrm{C}$ for $5 \mathrm{~h}$, then cooled to room temperature, diluted with EtOAc and washed with $1 \mathrm{~N} \mathrm{HCl}(\mathrm{aq})$. The layers were separated and the aqueous layer was extracted with EtOAc. The combined organic layers were dried, filtered and concentrated under reduced pressure. The crude material was chromatographed on $\mathrm{SiO}_{2}$ (3-15\% $\mathrm{Et}_{2} \mathrm{O} / \mathrm{CH}_{2} \mathrm{Cl}_{2}$ doped with $1 \% \mathrm{MeOH}$ as eluent) to afford $0.245 \mathrm{~g}(85 \%)$ of acid 3 as a white foam. IR (ZnSe): 3318, 2954, 2840, 1736, 1679, 1605, 1507, 1438, 1262 , 1156, 1140, 1082, $1021 \mathrm{~cm}^{-1} .{ }^{1} \mathrm{H}$ NMR $\left(400 \mathrm{MHz}, \mathrm{CDCl}_{3}\right): \delta 7.81(\mathrm{~d}, 1 \mathrm{H}, J=15.9 \mathrm{~Hz}$, $\left.\mathrm{CH}=\mathrm{CHCO}_{2} \mathrm{H}\right), 7.23(\mathrm{~d}, 1 \mathrm{H}, J=8.6 \mathrm{~Hz}, \operatorname{ArH}), 6.96-6.81(\mathrm{~m}, 4 \mathrm{H}, \operatorname{ArH~x~4}), 6.27(\mathrm{~d}, 1 \mathrm{H}$, $\left.J=15.9 \mathrm{~Hz}, \mathrm{CH}=\mathrm{CHCO}_{2} \mathrm{H}\right), 6.04(\mathrm{~d}, 1 \mathrm{H}, J=5.7 \mathrm{~Hz}, \operatorname{ArOCHAr}), 4.49(\mathrm{~d}, 1 \mathrm{H}, J=5.7 \mathrm{~Hz}$, $\left.\mathrm{ArCHCO}_{2} \mathrm{CH}_{3}\right), 3.93$ (s, 3H, OCH$\left.)_{3}\right), 3.85$ (s, 6H, OCH $\mathrm{OH}_{3}$ ), $3.79\left(\mathrm{~s}, 3 \mathrm{H}, \mathrm{CO}_{2} \mathrm{CH}_{3}\right) .{ }^{13} \mathrm{C}$ NMR (100 MHz, $\left.\mathrm{CDCl}_{3}\right): \delta 172.3,171.7,149.2,149.2,148.4,146.5,143.2,132.2,125.2$, $124.0,120.8,118.0,116.6,112.9,111.1,108.7,87.4,56.1,55.9,55.9,55.9,52.8 .[\alpha]_{D}(c$ 0.50, $\left.\mathrm{CH}_{2} \mathrm{Cl}_{2}\right)+168.4^{\mathrm{o}}$. $\mathrm{FAB}(+)-\mathrm{HRMS}$ Calcd. for $\mathrm{C}_{22} \mathrm{H}_{22} \mathrm{O}_{8}[\mathrm{M}]^{+}$: 414.1315. Found: 414.1312. 

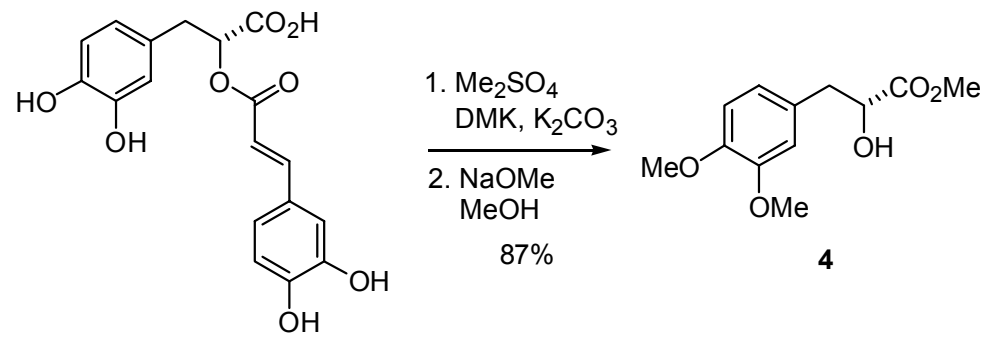

3-(3,4-Dimethoxy-phenyl)-2-hydroxy-propionic acid methyl ester (4). To a 1 L round bottom flask equipped with a magnetic stir bar and reflux condenser was added rosmarinic acid (3.08 g, $8.55 \mathrm{mmol}, 1.0$ equiv), acetone $(400 \mathrm{~mL})$, dimethyl sulfate (10.78 g, $85.48 \mathrm{mmol}, 10.0$ equiv) and potassium carbonate $(11.81 \mathrm{~g}, 85.48 \mathrm{mmol}, 10.0$ equiv). The reaction mixture was heated at reflux for $14 \mathrm{~h}$, cooled to room temperature, and diluted with $\mathrm{Et}_{2} \mathrm{O}$ and saturated aqueous $\mathrm{NH}_{4} \mathrm{Cl}$ solution. The layers were separated and the aqueous layer was extracted with $\mathrm{Et}_{2} \mathrm{O}$. The combined organic layers were washed with brine, dried, filtered and concentrated under reduced pressure. The crude material was chromatographed on $\mathrm{SiO}_{2}\left(10-40 \% \mathrm{Et}_{2} \mathrm{O} /\right.$ benzene as eluent) to afford pentamethyl rosmarinic acid as a light yellow foam. IR (ZnSe): 3003, 2950, 2934, 2835, $1753,1708,1630,1593,1503,1462,1422,1258,1230,1131,1029,976,805 \mathrm{~cm}^{-1} \cdot{ }^{1} \mathrm{H}$ NMR $\left(400 \mathrm{MHz}, \mathrm{CDCl}_{3}\right): \delta 7.58\left(\mathrm{~d}, 1 \mathrm{H}, J=15.9 \mathrm{~Hz}, \mathrm{CH}=\mathrm{CHCO}_{2} \mathrm{CH}\right), 7.00(\mathrm{~d}, 1 \mathrm{H}, J=$ $8.3 \mathrm{~Hz}, \operatorname{ArH}), 6.96$ (s, 1H, ArH), $6.77(\mathrm{~d}, 1 \mathrm{H}, J=8.3 \mathrm{~Hz}, \operatorname{ArH}), 6.73-6.72(3 \mathrm{H}, \mathrm{m}, \operatorname{ArH~x}$ 3), $6.27\left(\mathrm{~d}, 1 \mathrm{H}, J=15.9 \mathrm{~Hz}, \mathrm{CH}=\mathrm{CHCO}_{2} \mathrm{CH}\right), 5.29(\mathrm{dd}, 1 \mathrm{H}, J=5.0,7.9 \mathrm{~Hz}$, $\left.\mathrm{CHCO}_{2} \mathrm{CH}_{3}\right), 3.81\left(\mathrm{~s}, 3 \mathrm{H}, \mathrm{OCH}_{3}\right), 3.81\left(\mathrm{~s}, 3 \mathrm{H}, \mathrm{OCH}_{3}\right), 3.78\left(\mathrm{~s}, 3 \mathrm{H}, \mathrm{OCH}_{3}\right), 3.76(\mathrm{~s}, 3 \mathrm{H}$, $\left.\mathrm{OCH}_{3}\right), 3.66\left(\mathrm{~s}, 3 \mathrm{H}, \mathrm{CO}_{2} \mathrm{CH}_{3}\right), 3.14-3.04\left(\mathrm{~m}, 2 \mathrm{H}, \mathrm{ArCH}_{2} \mathrm{CHCO}_{2} \mathrm{CH}_{3}\right) .{ }^{13} \mathrm{C} \mathrm{NMR}(100$ $\left.\mathrm{MHz}, \mathrm{CDCl}_{3}\right): \delta 170.0,165.9,151.0,148.9,148.4,147.7,145.7,128.1,126.7,122.6$ $121.1,114.2,112.2,110.9,110.7,109.3,72.7,55.6,55.5,55.5,55.5,52.0,36.8 .[\alpha]_{D}(c$ 1.60, $\left.\mathrm{CH}_{2} \mathrm{Cl}_{2}\right)+40.7^{\circ}$. $\mathrm{FAB}(+)-\mathrm{HRMS}$ Calcd. for $\mathrm{C}_{23} \mathrm{H}_{26} \mathrm{O}_{8}[\mathrm{M}]^{+}$: 430.1628. Found: 
430.1628. Pentamethyl rosmarinic acid was dissolved in $\mathrm{MeOH}(40 \mathrm{~mL})$ and $\mathrm{NaOMe}$ ( $0.462 \mathrm{~g}, 8.55 \mathrm{mmol}, 1.0$ equiv) was added. The reaction mixture was stirred at room temperature for $2 \mathrm{~h}$. Saturated aqueous $\mathrm{NH}_{4} \mathrm{Cl}$ solution was added and the mixture was diluted with EtOAc. The layers were separated and the aqueous layer was extracted with EtOAc. The combined organic layers were dried, filtered and concentrated under reduced pressure. The crude material was chromatographed on $\mathrm{SiO}_{2}(1-10 \%$ $\mathrm{Et}_{2} \mathrm{O} / \mathrm{CH}_{2} \mathrm{Cl}_{2}$ as eluent) to afford $1.79 \mathrm{~g}$ ( $87 \%$ for two steps) of alcohol 4 . IR ( $\left.\mathrm{ZnSe}\right)$ : $3387,3007,2987,2942,2905,2831,1720,1593,1516,1417,1262,1205,1156,1140$, 1095, 1025, 964, 858, $784 \mathrm{~cm}^{-1} .{ }^{1} \mathrm{H}$ NMR (300 MHz, $\left.\mathrm{CDCl}_{3}\right): \delta 6.69(\mathrm{~m}, 3 \mathrm{H}, \mathrm{ArH})$, 4.35-4.27 (m, 1H, CHOH), 3.73 (s, 3H, OCH $), 3.72\left(\mathrm{~s}, 3 \mathrm{H}, \mathrm{OCH}_{3}\right), 3.63(\mathrm{~s}, 3 \mathrm{H}$, $\left.\mathrm{CO}_{2} \mathrm{CH}_{3}\right), 3.18-3.11(\mathrm{~m}, 1 \mathrm{H}, \mathrm{OH}), 2.94(\mathrm{dd}, 1 \mathrm{H}, J=4.4,14.0 \mathrm{~Hz}$, one of $\left.\operatorname{ArCH} \mathbf{H}_{2} \mathrm{CH}(\mathrm{OH}) \mathrm{CO}_{2} \mathrm{CH}_{3}\right), 2.79\left(\mathrm{dd}, 1 \mathrm{H}, J=6.9,14.0 \mathrm{~Hz}\right.$, one of $\left.\operatorname{ArCH} \mathbf{H}_{2} \mathrm{CH}(\mathrm{OH}) \mathrm{CO}_{2} \mathrm{CH}_{3}\right)$.

${ }^{13} \mathrm{C} \mathrm{NMR}\left(75 \mathrm{MHz}, \mathrm{CDCl}_{3}\right): \delta 174.1,148.3,147.5,128.6,121.1,112.3,110.7,71.1$, 55.3, 55.3, 51.8, 39.6. $[\alpha]_{D}\left(c 0.67, \mathrm{CH}_{2} \mathrm{Cl}_{2}\right)+10.6^{\circ} . \mathrm{FAB}(+)-\mathrm{HRMS}$ Calcd. for $\mathrm{C}_{12} \mathrm{H}_{16} \mathrm{O}_{5}[\mathrm{M}]^{+}:$240.0998. Found: 240.0998. Melting point: 53-54 ${ }^{\circ} \mathrm{C}$. 


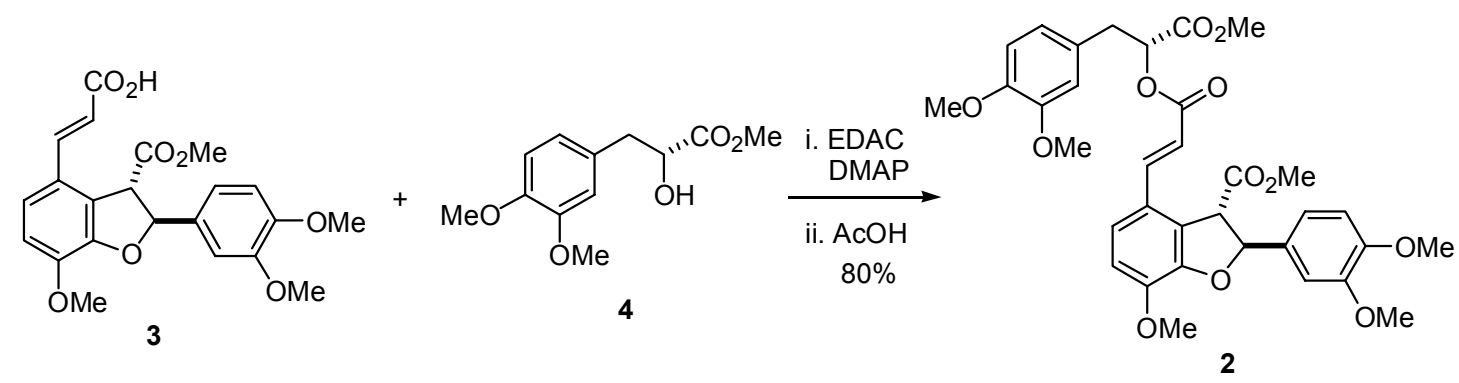

\section{2-(3,4-Dimethoxy-phenyl)-4-\{2-[2-(3,4-dimethoxy-phenyl)-1-methoxycarbonyl-} ethoxycarbonyl]-vinyl\}-7-methoxy-2,3-dihydro-benzofuran-3-carboxylic acid methyl ester (2). To a $50 \mathrm{~mL}$ round bottom flask equipped with a magnetic stir bar and septum was added $N$-(3-dimethylaminopropyl)- $N$ '-ethylcarbodiimide hydrochloride (EDC) (2.54 g, $13.3 \mathrm{mmol}$, 5.4 equiv), 4-dimethylaminopyridine (DMAP) (1.62 g, $13.3 \mathrm{mmol}, 5.4$ equiv), acid 3 (1.02 g, 2.46 mmol, 1.0 equiv), alcohol 4 (0.96 g, 3.98 mmol, 1.6 equiv) and $\mathrm{CHCl}_{3}(15 \mathrm{~mL})$, and the reaction mixture was stirred at room temperature for $2.5 \mathrm{~d}$. Acetic acid $(0.5 \mathrm{~mL})$ was added and the reaction mixture was stirred for 10 min to convert excess alcohol 4 to a more easily separable acetate. The mixture was washed with saturated aqueous $\mathrm{NH}_{4} \mathrm{Cl}$ solution, diluted with $\mathrm{Et}_{2} \mathrm{O}$, and the layers were separated. The aqueous layer was extracted with $\mathrm{Et}_{2} \mathrm{O}$, and the combined organic layers were dried, filtered and concentrated under reduced pressure. The crude material was chromatographed on $\mathrm{SiO}_{2}(10-90 \%$ EtOAc/hexanes as eluent) to afford $1.25 \mathrm{~g}(80 \%)$ of ester 2 as a white foam. IR (ZnSe): 3003, 2958, 2933, 2831, 1732, 1712, 1610, 1511, $1434,1258,1234,1152,1140,1078,1021,800,760 \mathrm{~cm}^{-1} .{ }^{1} \mathrm{H}$ NMR (400 MHz, $\left.\mathrm{CDCl}_{3}\right)$ : $\delta 7.71\left(\mathrm{~d}, 1 \mathrm{H}, J=15.9 \mathrm{~Hz}, \mathrm{CH}=\mathrm{CHCO}_{2} \mathrm{CH}\right), 7.17(\mathrm{~d}, 1 \mathrm{H}, J=8.5 \mathrm{~Hz}, \mathrm{ArH}), 6.89-6.85(\mathrm{~m}$, $3 \mathrm{H}, \operatorname{ArH} \times 3), 6.81(\mathrm{~d}, 1 \mathrm{H}, J=8.2 \mathrm{~Hz}, \operatorname{ArH}), 6.77-6.74(\mathrm{~m}, 3 \mathrm{H}, \operatorname{ArH} \times 3), 6.29(\mathrm{~d}, 1 \mathrm{H}, J$ $\left.=15.9 \mathrm{~Hz}, \mathrm{CH}=\mathrm{CHCO}{ }_{2} \mathrm{CH}\right), 6.01(\mathrm{~d}, 1 \mathrm{H}, J=5.6 \mathrm{~Hz}, \operatorname{ArOCHAr}), 5.31(\mathrm{dd}, 1 \mathrm{H}, J=4.8$, $\left.8.2 \mathrm{~Hz}, \mathrm{ArCH}_{2} \mathrm{CHCO}_{2} \mathrm{CH}_{3}\right), 4.45\left(\mathrm{~d}, 1 \mathrm{H}, J=5.6 \mathrm{~Hz}, \mathrm{ArCHCO}_{2} \mathrm{CH}_{3}\right), 3.91\left(\mathrm{~s}, 3 \mathrm{H}, \mathrm{OCH}_{3}\right)$, 
$3.84\left(\mathrm{~s}, 3 \mathrm{H}, \mathrm{OCH}_{3}\right), 3.83\left(\mathrm{~s}, 3 \mathrm{H}, \mathrm{OCH}_{3}\right), 3.82\left(\mathrm{~s}, 3 \mathrm{H}, \mathrm{OCH}_{3}\right), 3.81\left(\mathrm{~s}, 3 \mathrm{H}, \mathrm{OCH}_{3}\right), 3.71(\mathrm{~s}$, $\left.6 \mathrm{H}, \mathrm{CO}_{2} \mathrm{CH}_{3} \times 2\right), 3.16\left(\mathrm{dd}, 1 \mathrm{H}, J=4.7,14.3 \mathrm{~Hz}\right.$, one of $\left.\mathrm{ArCH}_{2} \mathrm{CHCO}_{2} \mathrm{CH}_{3}\right), 3.09$ (dd, $1 \mathrm{H}, J=8.2,14.3 \mathrm{~Hz}$, one of $\left.\mathrm{ArCH}_{2} \mathrm{CHCO}_{2} \mathrm{CH}_{3}\right) .{ }^{13} \mathrm{C} \mathrm{NMR}\left(100 \mathrm{MHz}, \mathrm{CDCl}_{3}\right): \delta$ 171.4, $170.0,165.8,149.0,149.0,148.5,148.2,147.8,146.1,142.2,132.1,128.8,128.1,124.7$, 124.1, 121.2, 120.6, 117.7, 116.2, 112.7, 112.2, 110.9, 110.9, 108.5, 87.2, 72.8, 55.9, 55.6, 55.6, 55.6, 55.5, 52.5, 52.0, 36.8. $[\alpha]_{D}\left(c 0.49, \mathrm{CH}_{2} \mathrm{Cl}_{2}\right)+134.5^{\circ}$. FAB(+)-HRMS Calcd. for $\mathrm{C}_{34} \mathrm{H}_{36} \mathrm{O}_{12}[\mathrm{M}]^{+}:$636.2207. Found: 636.2216 . 

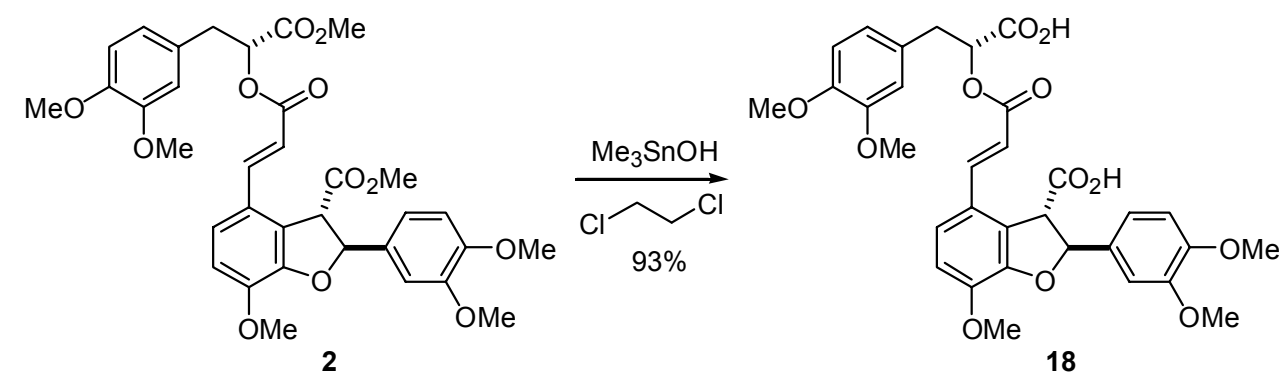

\section{4-\{2-[1-Carboxy-2-(3,4-dimethoxy-phenyl)-ethoxycarbonyl]-vinyl\}-2-(3,4-}

dimethoxy-phenyl)-7-methoxy-2,3-dihydro-benzofuran-3-carboxylic acid (21). To a

$100 \mathrm{~mL}$ round bottom flask equipped with a magnetic stir bar and septum was added heptamethyl lithospermic acid 2 (0.643 g, $1.01 \mathrm{mmol}, 1.0$ equiv), $\mathrm{Me}_{3} \mathrm{SnOH}$ (0.913 g, $5.05 \mathrm{mmol}, 5.0$ equiv) and 1,2-dichloroethane $(50 \mathrm{~mL})$. The reaction mixture was stirred at $80{ }^{\circ} \mathrm{C}$ for $21 \mathrm{~h}$. The flask was then cooled to room temperature, and the mixture was diluted with $1 \mathrm{~N} \mathrm{HCl}(\mathrm{aq})$ and EtOAc. The layers were separated, and the aqueous layer was extracted with EtOAc. The combined organic layers were dried, filtered and concentrated under reduced pressure. The crude material was chromatographed on $\mathrm{SiO}_{2}$ (5-50\% acetone $/ \mathrm{CH}_{2} \mathrm{Cl}_{2}$ doped with $0.5 \% \mathrm{AcOH}$ as eluent) to afford $0.573 \mathrm{~g}(93 \%)$ of diacid 18 as a white foam. IR (ZnSe): 3514, 3240, 3007, 2933, 2835, 1708, 1601, 1511, 1434, 1266, 1234, 1156, 1140, 1082, 1025, 805, $764 \mathrm{~cm}^{-1} .{ }^{1} \mathrm{H}$ NMR (400 MHz, $\left.\mathrm{CD}_{3} \mathrm{COCD}_{3}\right): \delta 7.86\left(\mathrm{~d}, 1 \mathrm{H}, J=16.0 \mathrm{~Hz}, \mathrm{CH}=\mathrm{CHCO}_{2} \mathrm{CH}\right), 7.37(\mathrm{~d}, 1 \mathrm{H}, J=8.6 \mathrm{~Hz}$, $\operatorname{ArH}), 7.08(\mathrm{~s}, 1 \mathrm{H}, \operatorname{ArH}), 7.02(\mathrm{~d}, 1 \mathrm{H}, J=8.6 \mathrm{~Hz}, \operatorname{ArH}), 6.98-6.82(\mathrm{~m}, 5 \mathrm{H}, \operatorname{ArH} \times \mathrm{x})$, $6.44\left(\mathrm{~d}, 1 \mathrm{H}, J=15.9 \mathrm{~Hz}, \mathrm{CH}=\mathrm{CHCO}_{2} \mathrm{CH}\right), 6.08(\mathrm{~d}, 1 \mathrm{H}, J=5.1 \mathrm{~Hz}, \operatorname{ArOCHAr}), 5.27$ (dd, $\left.1 \mathrm{H}, J=4.1,8.3 \mathrm{~Hz}, \mathrm{ArCH}_{2} \mathrm{CHCO}_{2} \mathrm{H}\right), 4.61\left(\mathrm{~d}, 1 \mathrm{H}, J=5.1 \mathrm{~Hz}, \operatorname{ArCHCO}_{2} \mathrm{H}\right), 3.91(\mathrm{~s}, 3 \mathrm{H}$, $\left.\mathrm{OCH}_{3}\right), 3.78\left(\mathrm{~s}, 6 \mathrm{H}, \mathrm{OCH}_{3} \times 2\right), 3.77\left(\mathrm{~s}, 3 \mathrm{H}, \mathrm{OCH}_{3}\right), 3.72\left(\mathrm{~s}, 3 \mathrm{H}, \mathrm{OCH} \mathbf{H}_{3}\right), 3.20$ (dd, 1H, $J$ $=4.0,14.3 \mathrm{~Hz}$, one of $\left.\mathrm{ArCH}_{2} \mathrm{CHCO}_{2} \mathrm{H}\right), 3.11(\mathrm{dd}, 1 \mathrm{H}, J=8.4,14.2 \mathrm{~Hz}$, one of $\left.\mathrm{ArCH}_{2} \mathrm{CHCO}_{2} \mathrm{H}\right) .{ }^{13} \mathrm{C} \mathrm{NMR}\left(100 \mathrm{MHz}, \mathrm{CD}_{3} \mathrm{COCD}_{3}\right): \delta 173.2,171.4,166.6,150.5$, 
150.1, 149.5, 149.3, 147.4, 143.1, 133.9, 130.0, 129.2, 127.0, 125.3, 122.6, 121.5, 118.9, $117.3,114.3,114.2,112.7,112.6,110.6,88.3,73.9,56.5,56.5,56.1,56.1,56.0,55.0$, 37.7. $[\alpha]_{D}\left(c 0.56, \mathrm{CH}_{2} \mathrm{Cl}_{2}\right)+173.8^{\circ}$. ES(-)-LRMS (negative ionization) Calcd. for $\mathrm{C}_{32} \mathrm{H}_{31} \mathrm{O}_{12}[\mathrm{M}-\mathrm{H}]$ : 607.2 Found: 607.1. 


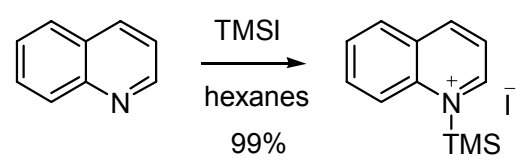

1-Trimethylsilylquinolinium iodide (TMSI-quinoline). Prepared according to a modified procedure of Brossi and Minamikawa. ${ }^{4}$ In a dry box, a solution of iodotrimethylsilane $(5.0 \mathrm{~mL}, 36.7 \mathrm{mmol}, 1.0$ equiv) in hexanes $(20 \mathrm{~mL})$ was slowly added to a solution of freshly distilled quinoline $(8.7 \mathrm{~mL}, 73.5 \mathrm{mmol}, 2.0$ equiv) in hexanes $(50 \mathrm{~mL})$ with vigorous stirring, and a yellow precipitate formed immediately. The mixture was filtered, and the solid was transferred to a sublimator and sublimed at $100{ }^{\circ} \mathrm{C}$ to afford $12 \mathrm{~g} \mathrm{(99 \% )} \mathrm{of} \mathrm{1-trimethylsilylquinolinium} \mathrm{iodide} \mathrm{as} \mathrm{a} \mathrm{yellow} \mathrm{solid}$ which was stored in a dry box. Melting point: $225-230{ }^{\circ} \mathrm{C} .{ }^{1} \mathrm{H} \mathrm{NMR}\left(400 \mathrm{MHz}, \mathrm{CDCl}_{3}\right)$ : $\delta 8.93(\mathrm{~d}, 1 \mathrm{H}, J=4.0 \mathrm{~Hz}, \operatorname{ArH}), 8.20(\mathrm{~d}, 1 \mathrm{H}, J=8.3 \mathrm{~Hz}, \operatorname{ArH}), 8.15(\mathrm{~d}, 1 \mathrm{H}, J=8.5 \mathrm{~Hz}$, $\operatorname{ArH}), 7.83(\mathrm{~d}, 1 \mathrm{H}, J=8.2 \mathrm{~Hz}, \operatorname{ArH}), 7.74(\mathrm{t}, 1 \mathrm{H}, J=7.6 \mathrm{~Hz}, \operatorname{ArH}), 7.56(\mathrm{t}, 1 \mathrm{H}, J=7.5$ $\mathrm{Hz}, \operatorname{ArH}), 7.43(\mathrm{dd}, 1 \mathrm{H}, J=4.3,8.3 \mathrm{~Hz}, \operatorname{ArH}), 0.79\left(\mathrm{~s}, 9 \mathrm{H}, \mathrm{Si}\left(\mathrm{CH}_{3}\right)_{3}\right) .{ }^{13} \mathrm{C} \mathrm{NMR}(100$ $\left.\mathrm{MHz}_{\mathrm{CDCl}}\right): \delta 149.9,147.6,136.7,129.8,128.9,128.3,127.8,126.8,121.1,5.5$.

\footnotetext{
${ }^{4}$ Minamikawa, J.; Brossi, A. Tetrahedron Lett. 1978, 34, 3085-3086.
} 

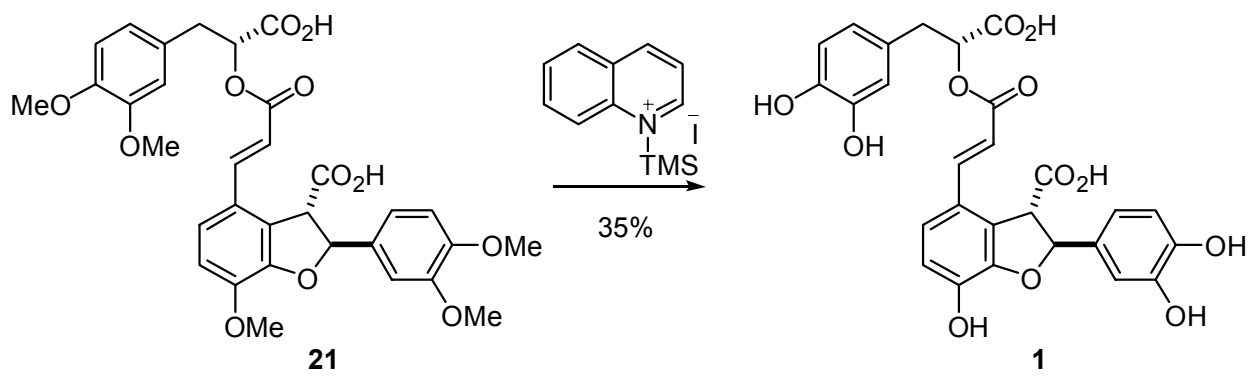

Lithospermic acid (1). In a dry box, diacid 18 (0.185 g, 0.304 mmol, 1.0 equiv) and 1trimethylsilylquinolinium iodide $(4.01 \mathrm{~g}, 12.2 \mathrm{mmol}, 40.0$ equiv) were added to a $20 \mathrm{~mL}$ sealed tube equipped with a magnetic stir bar. The reaction vessel was sealed and heated to $104{ }^{\circ} \mathrm{C}$ for $5 \mathrm{~h}$. The reaction mixture was then cooled to room temperature, vented and $1 \mathrm{~N} \mathrm{HCl}$ (aq) was slowly added. The reaction mixture was diluted with EtOAc, shaken vigorously, and the layers were separated. The aqueous layer was extracted with EtOAc, and the combined organic layers were dried, filtered and concentrated under reduced pressure. The crude material was chromatographed on $\mathrm{SiO}_{2}\left(5-40 \% \mathrm{MeOH} / \mathrm{CH}_{2} \mathrm{Cl}_{2}\right.$ doped with $1 \% \mathrm{AcOH}$ as eluent) to afford a dark residue. This residue was further purified by preparative HPLC $\left(\mathrm{C}_{18}\right.$ column, 5-65\% $\mathrm{MeCN} / \mathrm{H}_{2} \mathrm{O}$ with $0.1 \%$ TFA over 90 $\min , 20 \mathrm{~mL} / \mathrm{min}$, monitoring $220 \mathrm{~nm}$, injecting with a minimal amount of $\mathrm{MeOH}$, collecting fractions eluted from $31.5 \mathrm{~min}$ through $33.0 \mathrm{~min}$ ). The collected fractions were extracted with EtOAc, dried, filtered and concentrated under reduced pressure to afford $57 \mathrm{mg}(35 \%)$ of lithospermic acid as a dark solid. Lyophilizing a solution of lithospermic acid from $\mathrm{H}_{2} \mathrm{O} / \mathrm{MeCN}$ mixtures liberates the product as a pale yellow powder. Melting point: $183-188^{\circ} \mathrm{C} . \mathrm{IR}(\mathrm{ZnSe}): 3334,2954,2921,2856,1691,1605,1507,1446,1364$, $1283,1266,1180,1111,1070,976,813 \mathrm{~cm}^{-1} \cdot{ }^{1} \mathrm{H}$ NMR $\left(400 \mathrm{MHz}, \mathrm{CD}_{3} \mathrm{COCD}_{3}\right): \delta 7.85$ $\left(\mathrm{d}, 1 \mathrm{H}, J=16.0 \mathrm{~Hz}, \mathrm{CH}=\mathrm{CHCO}_{2} \mathrm{CH}\right), 7.30(\mathrm{~d}, 1 \mathrm{H}, J=8.5 \mathrm{~Hz}, \operatorname{ArH}), 6.90(\mathrm{~d}, 1 \mathrm{H}, J=8.3$ $\mathrm{Hz}, \operatorname{ArH}), 6.89$ (m, 1H, ArH), $6.86(\mathrm{~d}, 1 \mathrm{H}, J=2.0 \mathrm{~Hz}, \operatorname{ArH}), 6.82$ (d, 1H, J=8.1 Hz, 
ArH), $6.81(\mathrm{dd}, 1 \mathrm{H}, J=1.8,8.1 \mathrm{~Hz}, \operatorname{ArH}), 6.73(\mathrm{~d}, 1 \mathrm{H}, J=8.0 \mathrm{~Hz}, \operatorname{ArH}), 6.67(\mathrm{dd}, 1 \mathrm{H}, J$ $=2.0,8.1 \mathrm{~Hz}, \mathrm{ArH}), 6.37\left(\mathrm{~d}, 1 \mathrm{H}, J=15.9 \mathrm{~Hz}, \mathrm{CH}=\mathrm{CHCO}_{2} \mathrm{H}\right), 5.98(\mathrm{~d}, 1 \mathrm{H}, J=4.6 \mathrm{~Hz}$, ArOCHAr), 5.18 (dd, $\left.1 \mathrm{H}, J=4.1,8.5 \mathrm{~Hz}, \mathrm{ArCH}_{2} \mathrm{CHCO}_{2} \mathrm{H}\right), 4.53(\mathrm{~d}, 1 \mathrm{H}, J=4.7 \mathrm{~Hz}$, $\left.\operatorname{ArCHCO}_{2} \mathrm{H}\right), 3.10\left(\mathrm{dd}, 1 \mathrm{H}, J=4.1,14.3 \mathrm{~Hz}\right.$, one of $\left.\mathrm{ArCH}_{2} \mathrm{CHCO}_{2} \mathrm{H}\right), 3.01(\mathrm{dd}, 1 \mathrm{H}, J=$ 8.6, 14.3, one of $\left.\mathrm{ArCH}_{2} \mathrm{CHCO}_{2} \mathrm{H}\right) .{ }^{13} \mathrm{C}$ NMR $\left(125 \mathrm{MHz}, \mathrm{CD}_{3} \mathrm{OD},{ }^{1} \mathrm{H}\right.$ coupled): $\delta 175.1$ (s), $173.5(\mathrm{~s}), 168.2(\mathrm{~s}), 148.8(\mathrm{~s}), 146.7(\mathrm{~s}), 146.6(\mathrm{~s}), 146.0(\mathrm{~s}), 145.3(\mathrm{~s}), 145.2(\mathrm{~s})$, 144.2 (d), 133.8 (s), 129.3 (s), 127.6 (s), 124.6 (s), 122.1 (d), 121.8 (d), 118.6 (d), 118.3 (d), 117.4 (d), 116.4 (d), 116.4 (d), 116.4 (d), 113.4 (d), 88.8 (d), 74.8 (d), 57.5 (d), 37.9 (t). $[\alpha]_{D}(c 0.69, \mathrm{MeOH})+136.9^{\circ}\left(\right.$ lit. $\left.+78^{\circ}\right) .^{5}$ ES(-)-LRMS (negative ionization) Calcd. for $\mathrm{C}_{27} \mathrm{H}_{21} \mathrm{O}_{12}[\mathrm{M}-\mathrm{H}]$ : $: 537.1$ Found: 537.0.

\footnotetext{
${ }^{5}$ Lin, Y.L.; Wang, C.N.; Shiao, Y.J.; Liu, T.Y.; Wang, W.Y. J. Chin. Chem. Soc. 2003, 50, 1079-1083.
} 


\section{Lithospermic acid comparison table}

\section{${ }^{1}$ H NMR data (acetone-d $\left.{ }_{6}\right)^{6}$}

\begin{tabular}{|ll|l|}
\hline & $\begin{array}{l}\text { Natural (220 MHz) } \\
\delta(\mathrm{m}, J \text { in Hz) }\end{array}$ & \multicolumn{1}{|c|}{$\begin{array}{c}\text { Synthetic }(400 \mathrm{MHz}) \\
(\mathrm{m}, J \text { in } \mathrm{Hz})\end{array}$} \\
\hline H-7 & $7.80(\mathrm{~d}, 16)$ & $7.85(\mathrm{~d}, 16.0)$ \\
H-6 & $7.21(\mathrm{~d}, 8)$ & $7.30(\mathrm{~d}, 8.5)$ \\
H-5 & $6.83(\mathrm{~d}, 8)$ & $6.90(\mathrm{~d}, 8.3)$ \\
H-13 & $6.82(\mathrm{~d}, 2)$ & $6.89(\mathrm{~s})$ \\
H-23 & $6.81(\mathrm{~d}, 2)$ & $6.86(\mathrm{~d}, 2.0)$ \\
H-26 & $6.77(\mathrm{~d}, 8)$ & $6.82(\mathrm{~d}, 8.1)$ \\
H-27 & $6.72(\mathrm{dd}, 2,8)$ & $6.81(\mathrm{dd}, 1.8,8.1)$ \\
H-16 & $6.69(\mathrm{~d}, 8)$ & $6.73(\mathrm{~d}, 8.0)$ \\
H-17 & $6.61(\mathrm{dd}, 2,8)$ & $6.67(\mathrm{dd}, 2.0,8.1)$ \\
H-8 & $6.32(\mathrm{~d}, 16)$ & $6.37(\mathrm{~d}, 15.9)$ \\
H-21 & $5.94(\mathrm{~d}, 4.8)$ & $5.98(\mathrm{~d}, 4.6)$ \\
H-10 & $5.15(\mathrm{dd}, 5,8)$ & $5.18(\mathrm{dd}, 4.1,8.5)$ \\
H-20 & $4.47(\mathrm{~d}, 4.8)$ & $4.53(\mathrm{~d}, 4.7)$ \\
H-11 & $3.03(\mathrm{q}, 5,14)$ & $3.10(\mathrm{dd}, 4.1,14.3)$ \\
H-11 & $3.03(\mathrm{q}, 8,14)$ & $3.01(\mathrm{dd}, 8.6,14.3)$ \\
\hline
\end{tabular}

\section{${ }^{13} \mathrm{C}$ NMR data $\left(\mathrm{CD}_{3} \mathrm{OD},{ }^{1} \mathrm{H} \text { coupled }\right)^{7}$}

\begin{tabular}{|c|c|c|}
\hline & $\begin{array}{l}\text { Natural }(125 \mathrm{MHz}) \\
\delta(\mathrm{m})\end{array}$ & $\begin{array}{c}\text { Synthetic (125 MHz) } \\
\delta(\mathrm{m})\end{array}$ \\
\hline C-28 & $175.0(\mathrm{~s})$ & $175.1(\mathrm{~s})$ \\
\hline C-29 & $173.4(\mathrm{~s})$ & $173.5(\mathrm{~s})$ \\
\hline C-9 & $168.1(\mathrm{~s})$ & $168.2(\mathrm{~s})$ \\
\hline C-3 & $148.6(\mathrm{~s})$ & $148.8(\mathrm{~s})$ \\
\hline$C-25$ & $146.4(\mathrm{~s})$ & $146.7(\mathrm{~s})$ \\
\hline C-24 & $146.3(\mathrm{~s})$ & $146.6(\mathrm{~s})$ \\
\hline C-15 & $145.8(\mathrm{~s})$ & $146.0(\mathrm{~s})$ \\
\hline C-14 & $144.9(\mathrm{~s})$ & $145.3(\mathrm{~s})$ \\
\hline C-4 & $144.9(\mathrm{~s})$ & $145.2(\mathrm{~s})$ \\
\hline C-7 & $143.0(\mathrm{~d})$ & 144.2 (d) \\
\hline $\mathrm{C}-22$ & $133.5(\mathrm{~s})$ & $133.8(\mathrm{~s})$ \\
\hline C-12 & $129.1(\mathrm{~s})$ & $129.3(\mathrm{~s})$ \\
\hline $\mathrm{C}-2$ & $127.3(\mathrm{~s})$ & $127.6(\mathrm{~s})$ \\
\hline$C-1$ & $124.4(\mathrm{~s})$ & $124.6(\mathrm{~s})$ \\
\hline C-27 & $121.9(\mathrm{~d})$ & 122.1 (d) \\
\hline C-6 & 120.9 (d) & $121.8(\mathrm{~d})$ \\
\hline C-17 & 118.3 (d) & 118.6 (d) \\
\hline C-5 & 118.2 (d) & 118.3 (d) \\
\hline C-23 & 117.4 (d) & 117.4 (d) \\
\hline C-8 & $116.4(\mathrm{~d})$ & 116.4 (d) \\
\hline C-26 & 116.3 (d) & 116.4 (d) \\
\hline C-16 & 116.2 (d) & 116.4 (d) \\
\hline C-13 & $113.3(\mathrm{~d})$ & 113.4 (d) \\
\hline C-20 & 88.6 (d) & 88.8 (d) \\
\hline$C-10$ & 74.7 (d) & 74.8 (d) \\
\hline C-21 & $57.2(d)$ & 57.5 (d) \\
\hline$C-11$ & $37.6(t)$ & $37.9(\mathrm{t})$ \\
\hline
\end{tabular}

\footnotetext{
${ }^{6}$ Natural ${ }^{1} \mathrm{H}-\mathrm{NMR}$ data have been reproduced from the reported isolation, see: Kelley, C.J.; Mahajan, J.R.; Brooks, L.C.; Neubert, L.A.; Breneman, W.R.; Carmack, M. J. Org. Chem. 1975, 40, 1804-1815.

${ }^{7}$ Natural ${ }^{13} \mathrm{C}-\mathrm{NMR}$ data have been reproduced from the reported isolation, see: Lin, Y.L.; Wang, C.N.; Shiao, Y.J.; Liu, T.Y.; Wang, W.Y. J. Chin. Chem. Soc. 2003, 50, 1079-1083.
} 


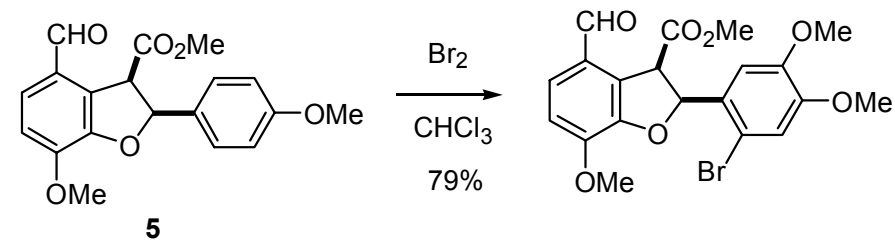

\section{2-(2-Bromo-4,5-dimethoxy-phenyl)-4-formyl-7-methoxy-2,3-dihydro-benzofuran-3-}

carboxylic acid methyl ester. To a $25 \mathrm{~mL}$ round bottom flask equipped with a magnetic stir bar and septum was added aldehyde $5\left(0.259 \mathrm{~g}, 0.696 \mathrm{mmol}, 1.0\right.$ equiv) and $\mathrm{CHCl}_{3}(7$ $\mathrm{mL})$. The flask was cooled to $0{ }^{\circ} \mathrm{C}$ in an ice water bath and $\mathrm{Br}_{2}(0.036 \mathrm{~mL}, 0.696 \mathrm{mmol}$, 1.0 equiv) was added. The mixture was stirred for $20 \mathrm{~min}$ followed by diluting with saturated aqueous sodium thiosulfate solution and $\mathrm{CH}_{2} \mathrm{Cl}_{2}$. The layers were separated and the aqueous layer was extracted with $\mathrm{CH}_{2} \mathrm{Cl}_{2}$. The combined organic layers were dried, filtered and concentrated under reduced pressure. The crude material was chromatographed on $\mathrm{SiO}_{2}\left(1-10 \% \mathrm{Et}_{2} \mathrm{O} / \mathrm{CH}_{2} \mathrm{Cl}_{2}\right.$ as eluent) to afford $0.246 \mathrm{~g}(79 \%)$ of the mono-brominated compound as a white foam. IR (ZnSe): 3007, 2970, 2946, 2835, 1712, $1740,1614,1581,1507,1438,1385,1283,1262,1201,1164,1176,1091,1025,800,768$ $\mathrm{cm}^{-1} .{ }^{1} \mathrm{H}$ NMR $\left(300 \mathrm{MHz}, \mathrm{CDCl}_{3}\right): \delta 9.85(\mathrm{~s}, 1 \mathrm{H}, \mathrm{CHO}), 7.43(\mathrm{~d}, 1 \mathrm{H}, J=8.4 \mathrm{~Hz}, \mathrm{ArH})$, 7.07-7.00 (m, 3H, ArH x 3), 6.27 (d, 1H, $J=9.6 \mathrm{~Hz}, \operatorname{ArOCHAr}), 5.03$ (d, 1H, $J=9.9 \mathrm{~Hz}$ $\left.\mathrm{ArCHCO}_{2} \mathrm{CH}_{3}\right), 4.00\left(\mathrm{~s}, 3 \mathrm{H}, \mathrm{OCH}_{3}\right), 3.88\left(\mathrm{~s}, 3 \mathrm{H}, \mathrm{OCH}_{3}\right), 3.82\left(\mathrm{~s}, 3 \mathrm{H}, \mathrm{OCH}_{3}\right), 3.22(\mathrm{~s}$, $\left.3 \mathrm{H}, \mathrm{CO}_{2} \mathrm{CH}_{3}\right) .{ }^{13} \mathrm{C}$ NMR $\left(75 \mathrm{MHz}, \mathrm{CDCl}_{3}\right): \delta 190.4,169.5,149.6,149.5,149.2,148.0$, $128.7,128.1,127.2,126.3,125.2,114.6,112.3,111.5,110.4,86.8,56.0,56.0,52.0,51.6$. $[\alpha]_{D}\left(c\right.$ 1.43, $\left.\mathrm{CH}_{2} \mathrm{Cl}_{2}\right)-174.9^{\circ}$. FAB(+)-HRMS Calcd. for $\mathrm{C}_{20} \mathrm{H}_{19} \mathrm{BrO}_{7} \mathrm{Li}[\mathrm{M}+\mathrm{Li}]^{+}: 457.0477$. Found: 457.0481. 


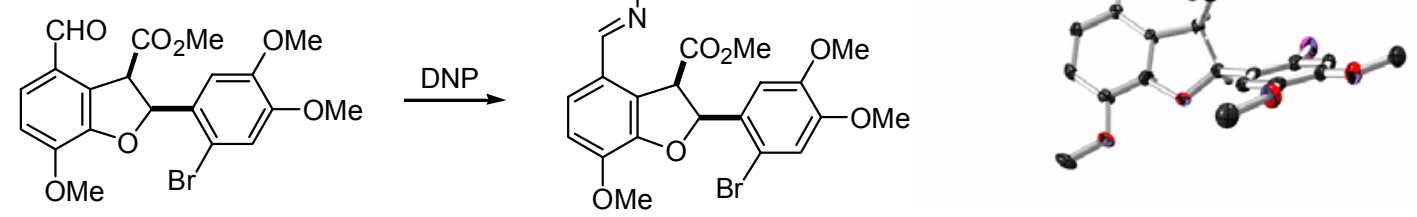

2-(2-Bromo-4,5-dimethoxy-phenyl)-4-[(2,4-dinitrophenyl)-hydrazonomethyl]-7methoxy-2,3-dihydro-benzofuran-3-carboxylic acid methyl ester. 2,4-

dinitrophenylhydrazine $(1.0 \mathrm{~g}, 5.0 \mathrm{mmol})$ was dissolved in concentrated sulfuric acid (5 $\mathrm{mL})$ and added to a flask containing $\mathrm{EtOH}(25 \mathrm{~mL})$ and $\mathrm{H}_{2} \mathrm{O}(7 \mathrm{~mL})$. A $2 \mathrm{~mL}$ portion of this solution was then added to a solution of bromide ( $0.098 \mathrm{~g}, 0.217 \mathrm{mmol})$ in $\mathrm{EtOH}$ (2 $\mathrm{mL}$ ). A red precipitate formed immediately, which was filtered through filter paper and crystallized from $\mathrm{CH}_{2} \mathrm{Cl}_{2} /$ pentane by vapor diffusion to afford $0.081 \mathrm{~g} \mathrm{(59 \% )}$ of the 2,4dinitrophenylhydrazone derivative as red needles. Melting point: $242-244{ }^{\circ} \mathrm{C}$. IR (ZnSe): 3277, 3117, 3003, 2950, 2840, 1736, 1610, 1585, 1499, 1438, 1332, 1283, 1262, 1201, 1136, 1082, 1025, 739. ${ }^{1} \mathrm{H}$ NMR (300 MHz, $\left.\mathrm{CDCl}_{3}\right): \delta 11.24$ (s, 1H, NH), $9.08(\mathrm{~d}$, $1 \mathrm{H}, J=2.2 \mathrm{~Hz}, \operatorname{ArH}), 8.31(\mathrm{dd}, 1 \mathrm{H}, J=2.6,9.6 \mathrm{~Hz}, \operatorname{ArH}), 8.08(\mathrm{~s}, 1 \mathrm{H}, \mathrm{CH}=\mathrm{N}), 7.97(\mathrm{~d}$, $1 \mathrm{H}, J=9.6 \mathrm{~Hz}, \operatorname{ArH}), 7.12-7.08(\mathrm{~m}, 3 \mathrm{H}, \operatorname{ArH} \times 3), 6.96(\mathrm{~d}, 1 \mathrm{H}, J=8.4 \mathrm{~Hz}, \operatorname{ArH}), 6.28(\mathrm{~d}$, $1 \mathrm{H}, J=9.4 \mathrm{~Hz}, \operatorname{ArOCHAr}), 5.07\left(\mathrm{~d}, 1 \mathrm{H}, J=9.4 \mathrm{~Hz}, \mathrm{ArCHCO}_{2} \mathrm{CH}_{3}\right), 3.99\left(\mathrm{~s}, 3 \mathrm{H}, \mathrm{OCH}_{3}\right)$, 3.90 (s, 3H, OCH $\left.\mathbf{H}_{3}\right), 3.84$ (s, 3H, OCH$), 3.18\left(\mathrm{~s}, 3 \mathrm{H}, \mathrm{CO}_{2} \mathrm{CH}_{3}\right) .{ }^{13} \mathrm{C} \mathrm{NMR}(75 \mathrm{MHz}$, $\left.\mathrm{CDCl}_{3}\right): \delta 169.5,153.7,149.8,149.4,148.3,148.0,147.2,144.3,138.0,129.9,129.1$, $127.3,125.8,123.3,122.6,116.6,114.8,112.5,112.2,110.4,86.7,56.2,56.1,56.0,53.4$, 51.7. $[\alpha]_{D}\left(c\right.$ 1.17, $\left.\mathrm{CH}_{2} \mathrm{Cl}_{2}\right)-43.8^{\circ}$. FAB(+)-HRMS Calcd. for $\mathrm{C}_{26} \mathrm{H}_{23} \mathrm{BrN}_{4} \mathrm{O}_{10}[\mathrm{M}]^{+}$: 630.0597. Found: 630.0595 . 
Total Synthesis of (+)-Lithospermic Acid by Asymmetric Intramolecular Alkylation via Catalytic C-H Bond Activation

\author{
Steven J. O’Malley, Kian L. Tan, Robert G. Bergman* and Jonathan A. Ellman* \\ Department of Chemistry, University of California-Berkeley, Berkeley, CA 94720 \\ Supporting Information (Spectral data)
}




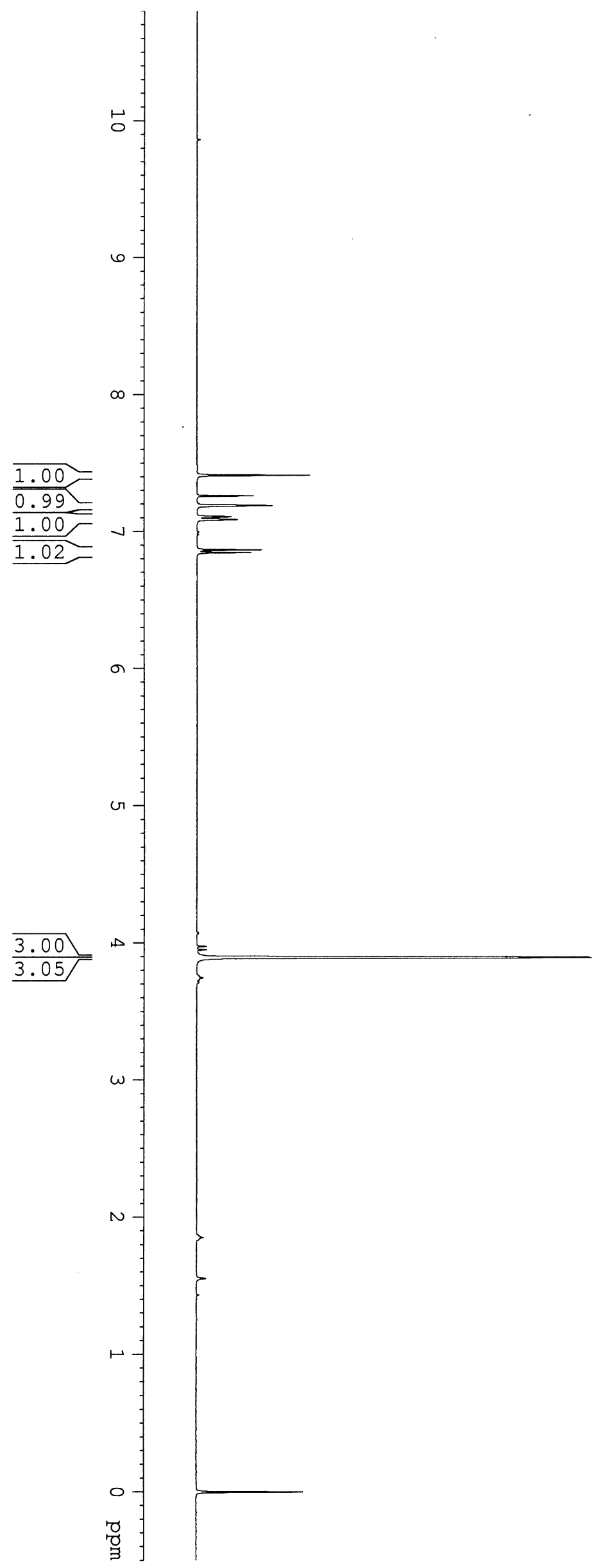

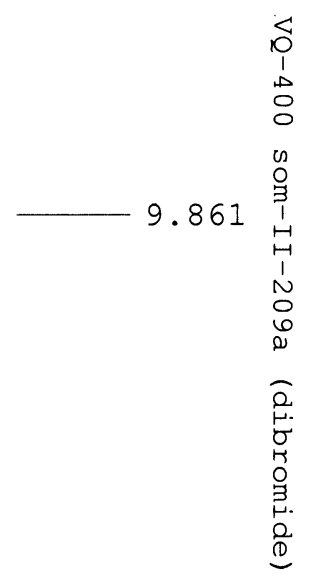
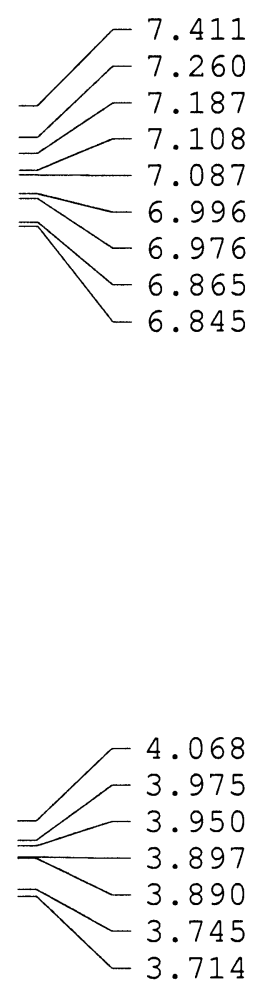

1.851

1.552

1.430

$-0.002$ 

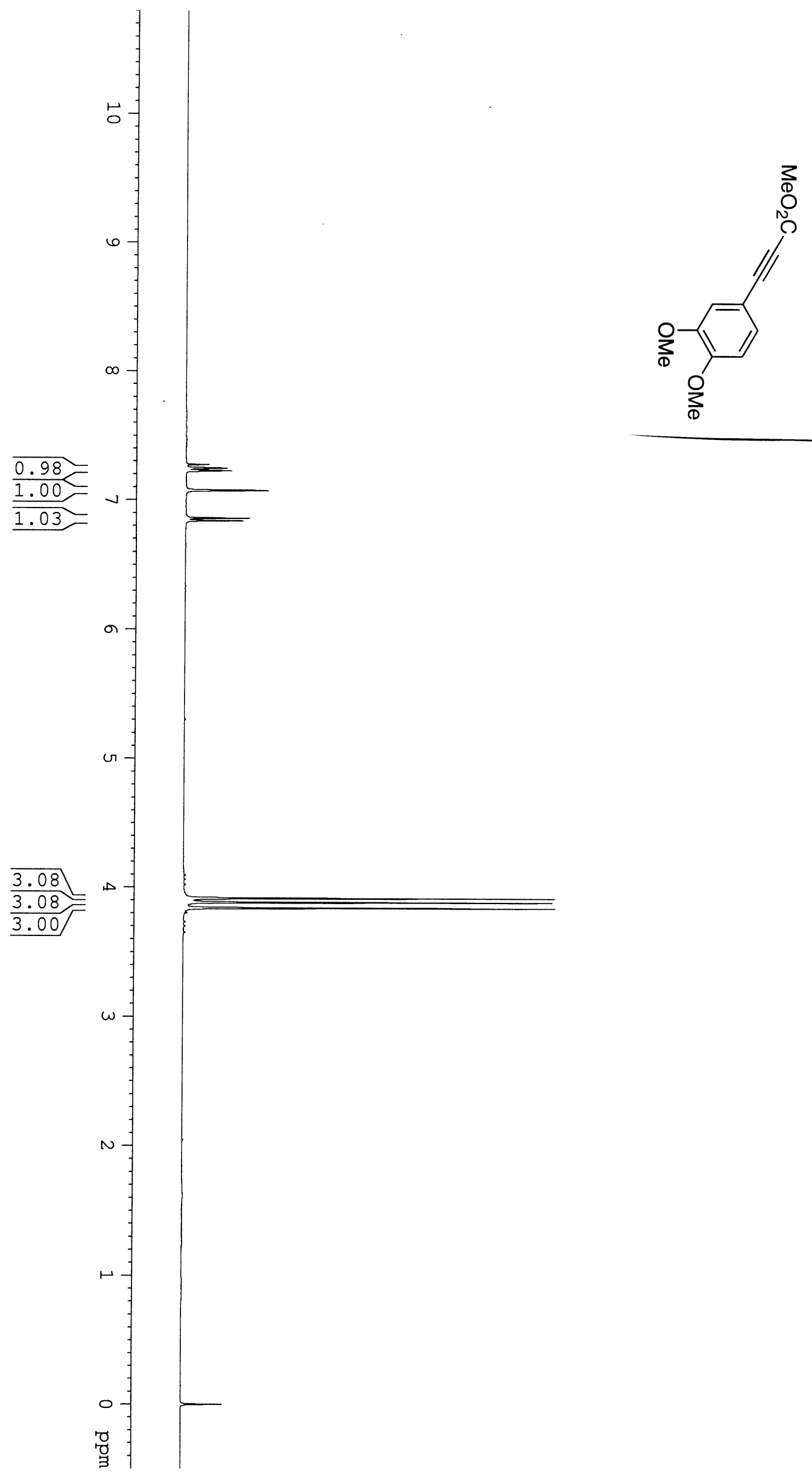

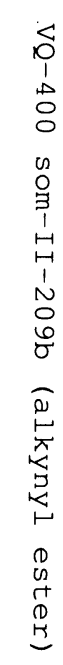
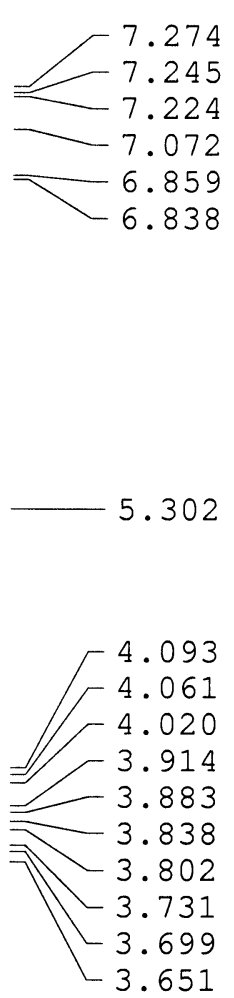

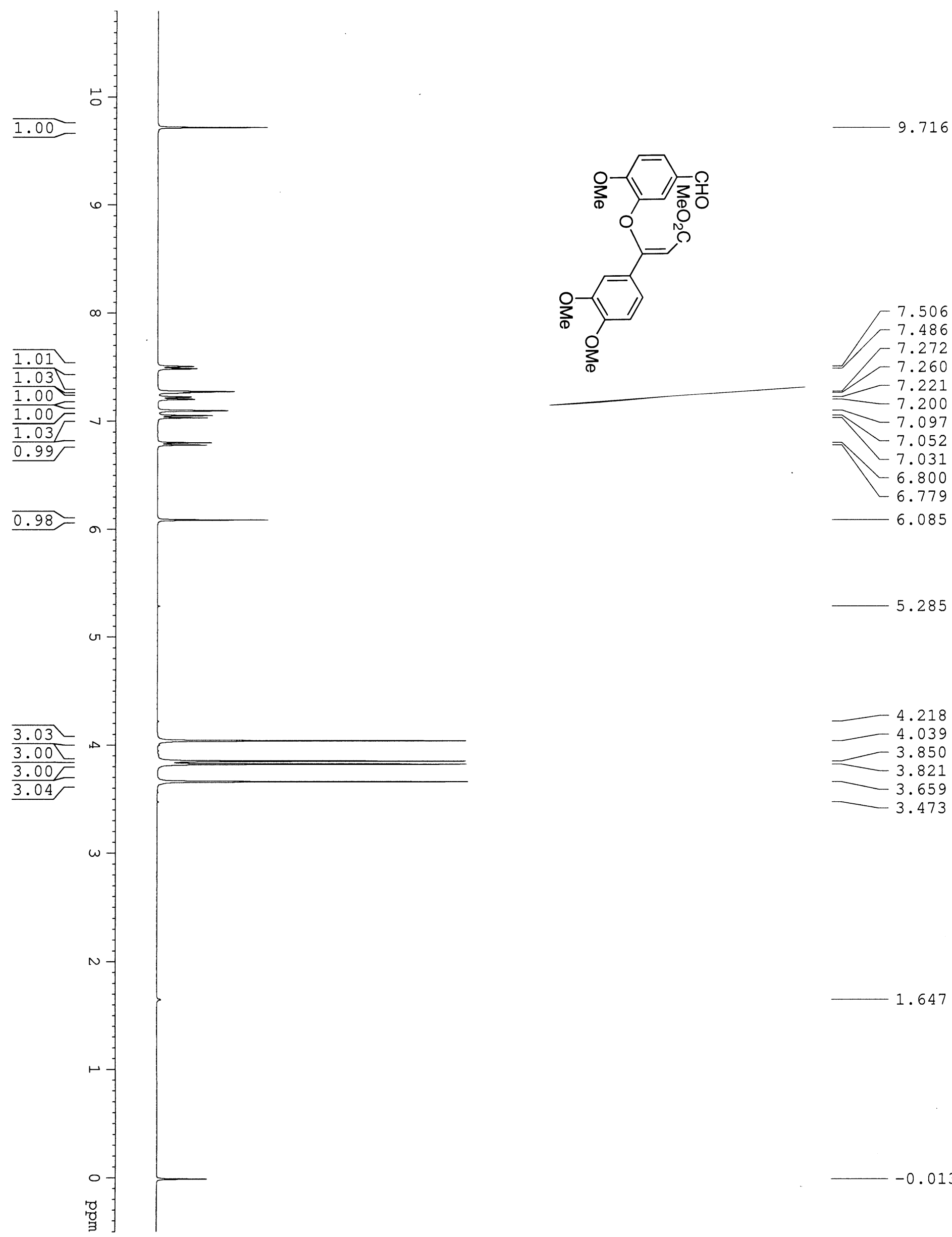

5.285

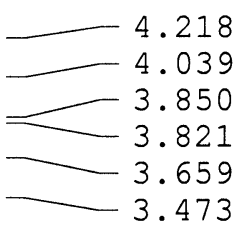




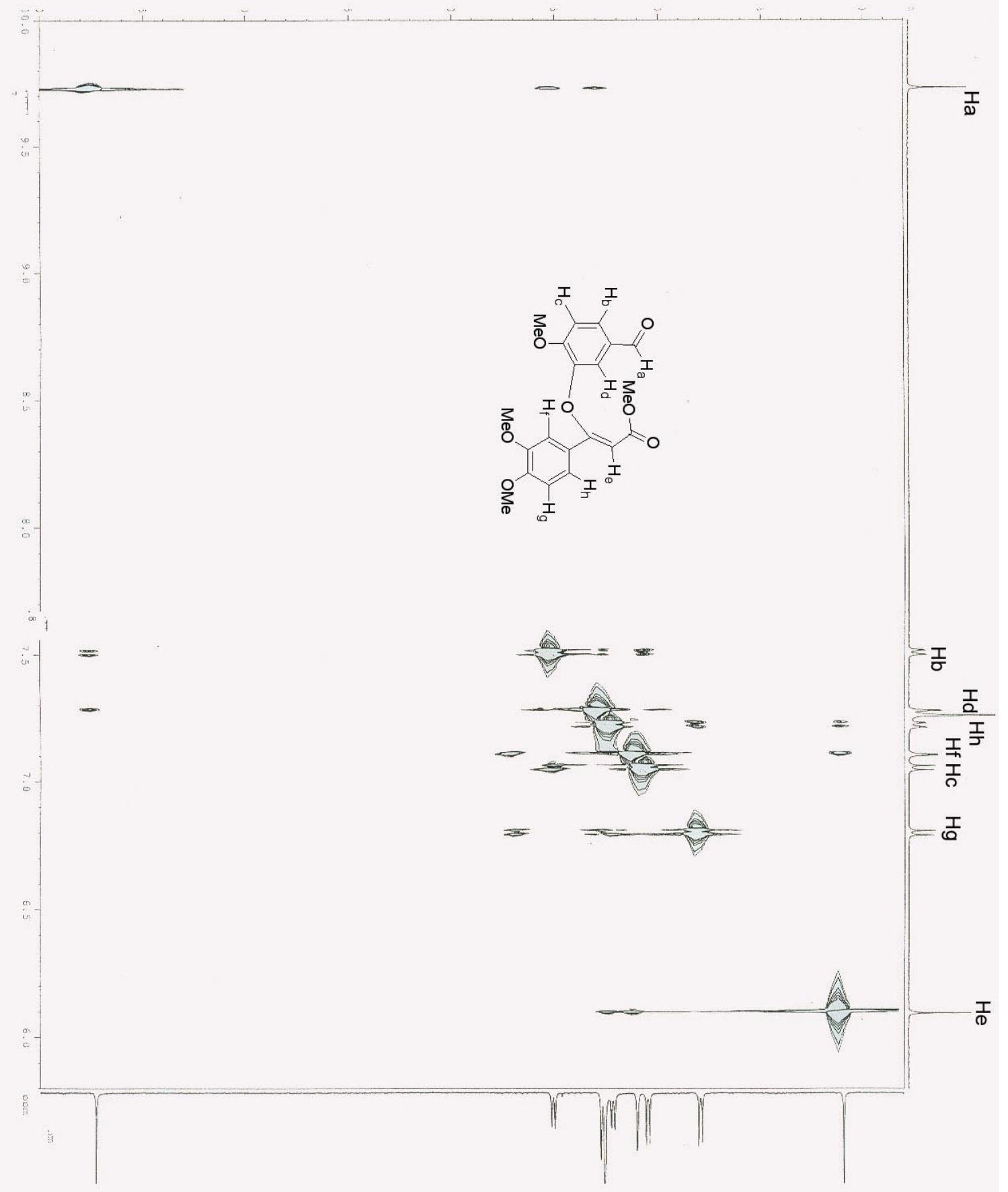

NOESY spectrum of (Z)-olefin 


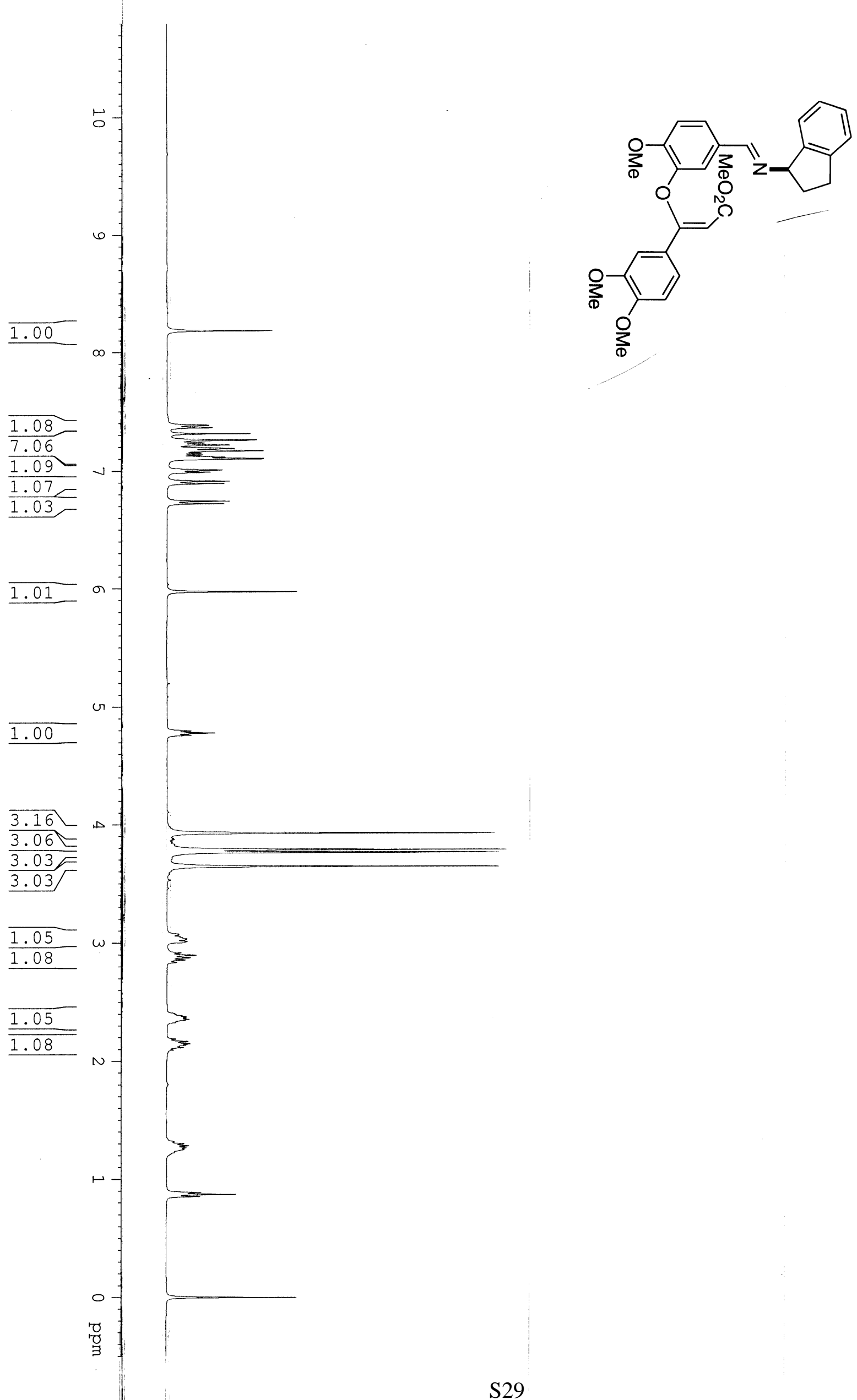

気 -2.377

1.316

$-1.300$

$\leftarrow 1.282$

1.265 

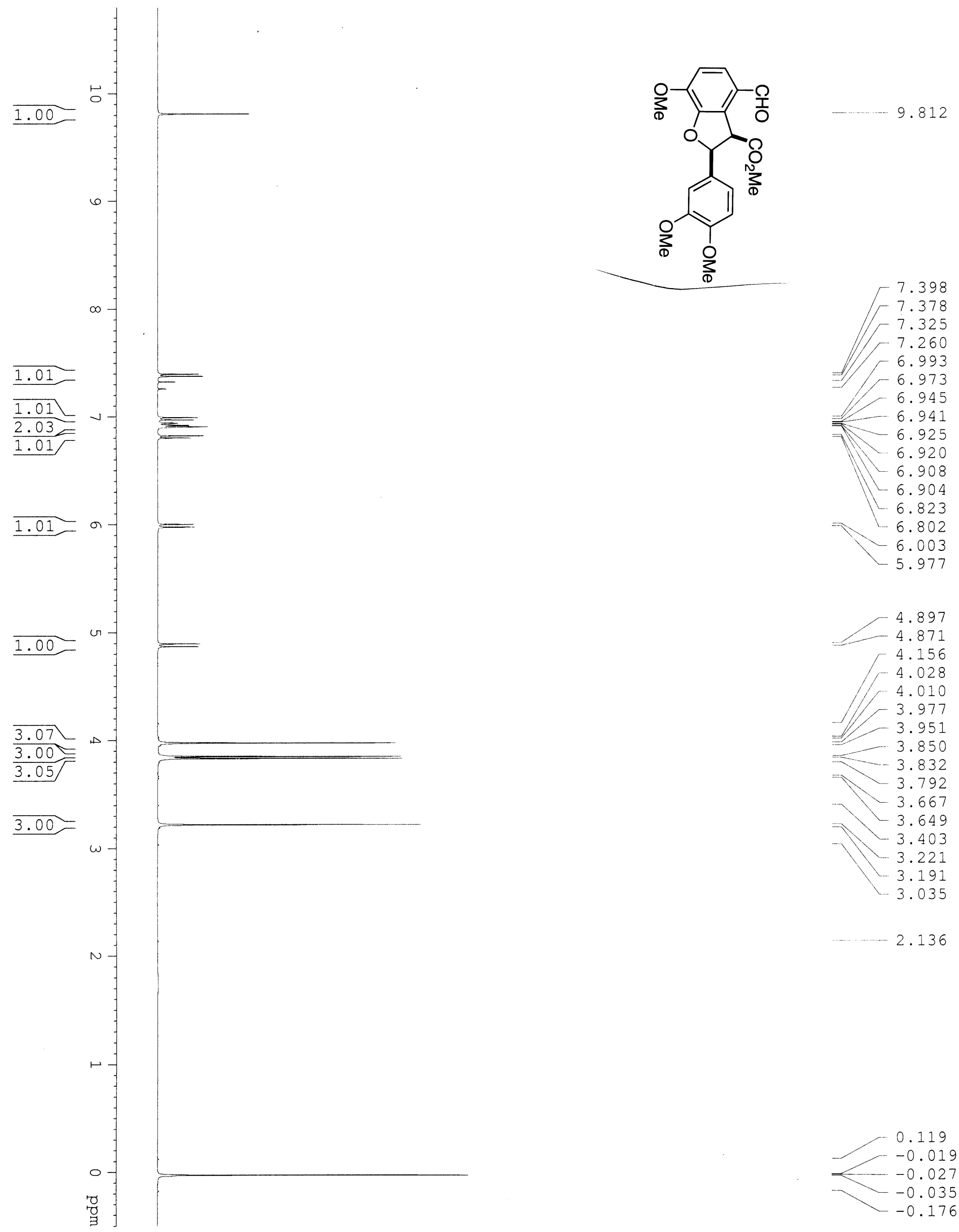

2.136 
<smiles>COc1ccc(C2Oc3c(OC)ccc(C=O)c3C2C(C)=O)cc1OC</smiles>

(士)

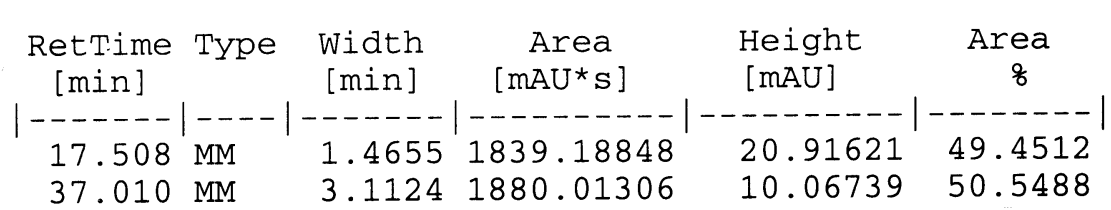

$37.010 \mathrm{MM}$

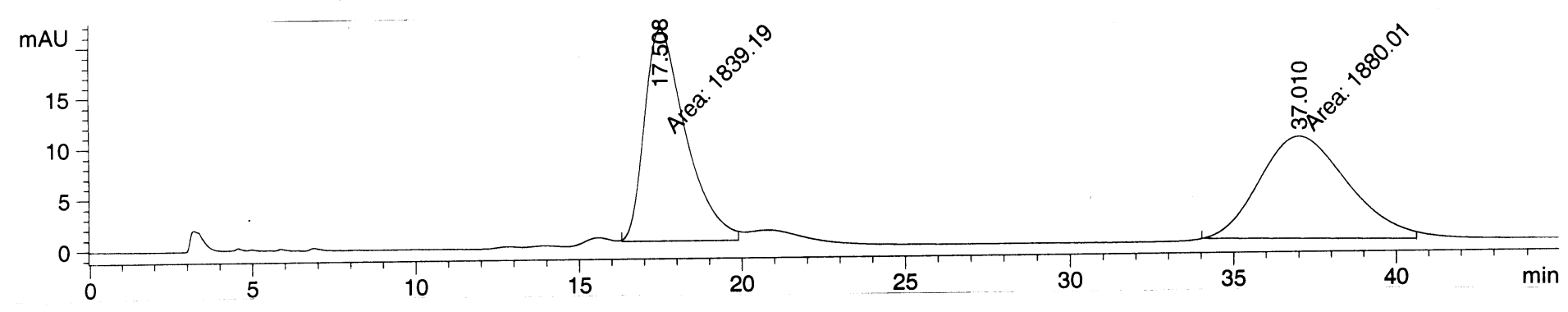<smiles>COc1ccc(C2Oc3c(OC)ccc(C=O)c3C2C(C)=O)cc1OC</smiles>

$73 \%$ ee

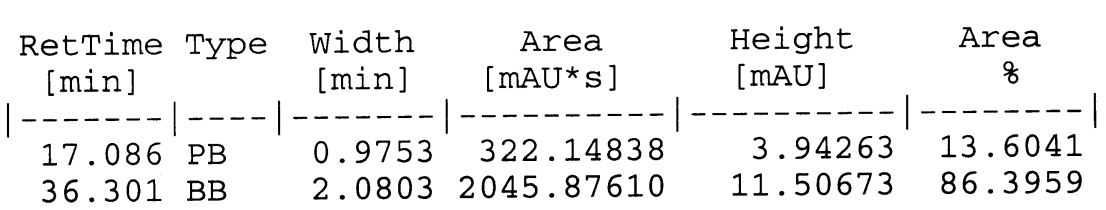

$36.301 \mathrm{BB}$

11.50673

86.3959

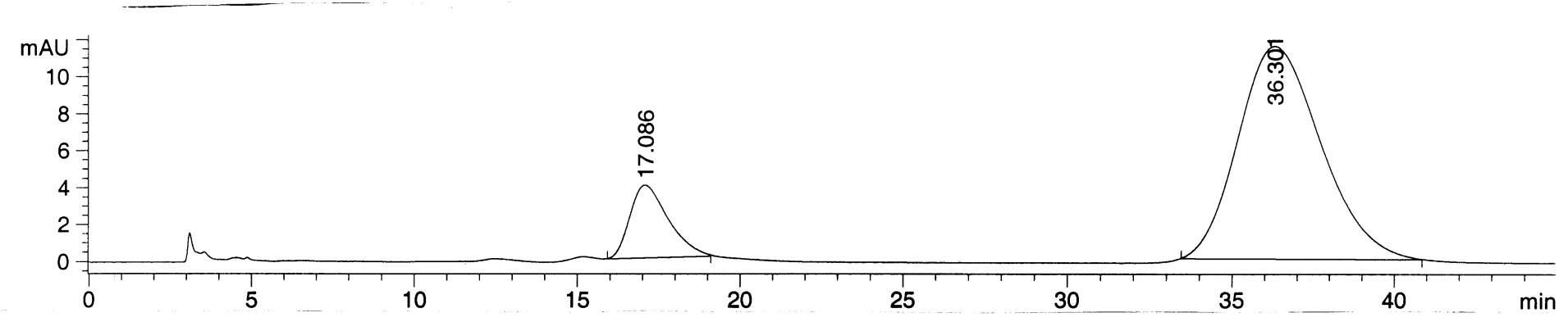<smiles>COc1ccc(C2Oc3c(OC)ccc(C=O)c3C2C(C)=O)cc1OC</smiles>

$>99 \%$ ee
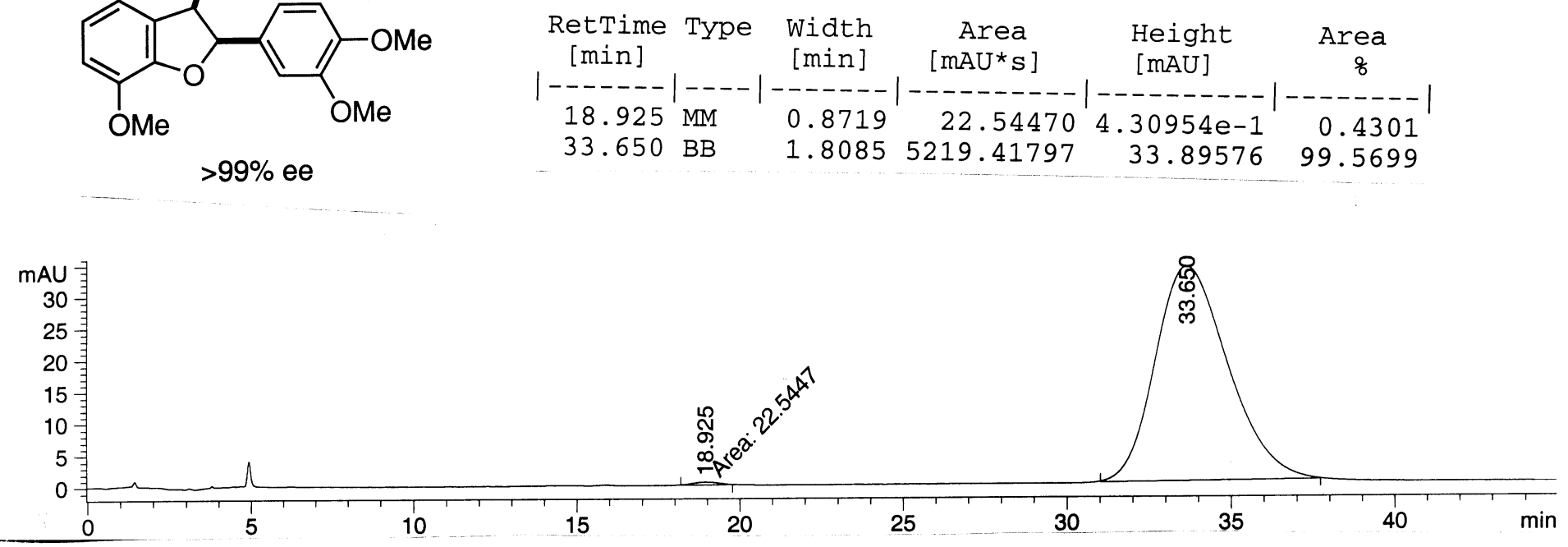

S31 

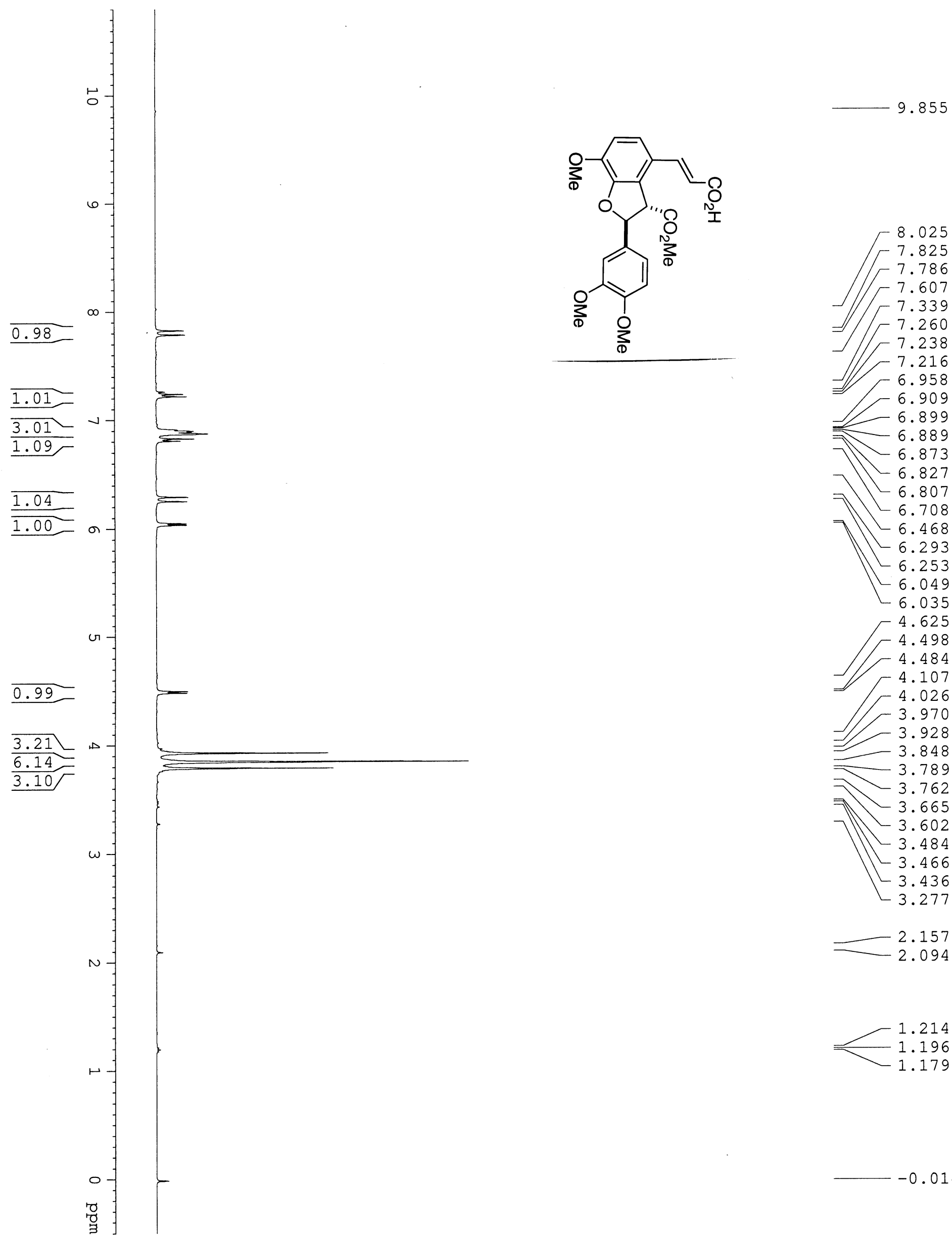

$\rrbracket-3.665$

$\square-3.602$

3.484

$-3.466$

$-3.436$

3.277

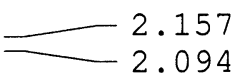

2.094

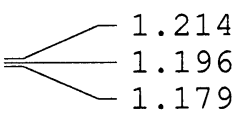

$-0.014$ 

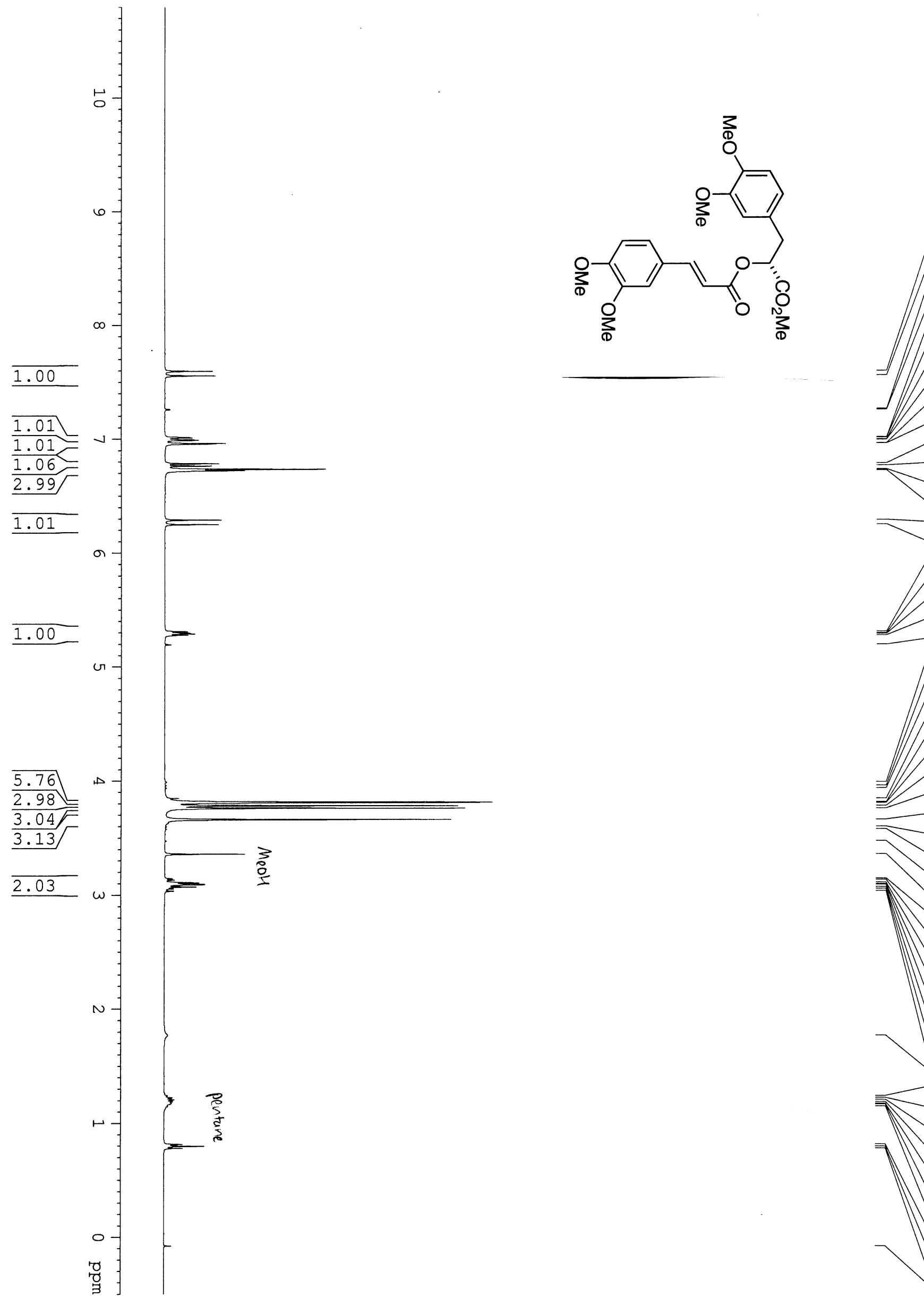

$-6.250$

5.310

5.297

$-5.290$

$\begin{array}{r}5.290 \\ -5.277 \\ \hline\end{array}$

$-5.195$

$-3.991$

- 3.959

$-3.937$

3.846
-3.813

3.813

$-3.809$

$-3.781$

-3.759
-3.660

3.660

-3.598
$-\quad 3.577$

$\checkmark 3.577$

$-3.473$

$\square .359$
-3.144

$-3.144$

$-3.131$

$-3.108$

3.095

3.091

$-3.071$

$-3.055$

$-3.035$

1.770

1.241

1.225

$-1.208$

$\approx\lfloor-1.189$

-1.173
1.165

$-1.148$

$-0.814$

0.796

0.778

$-0.078$ 

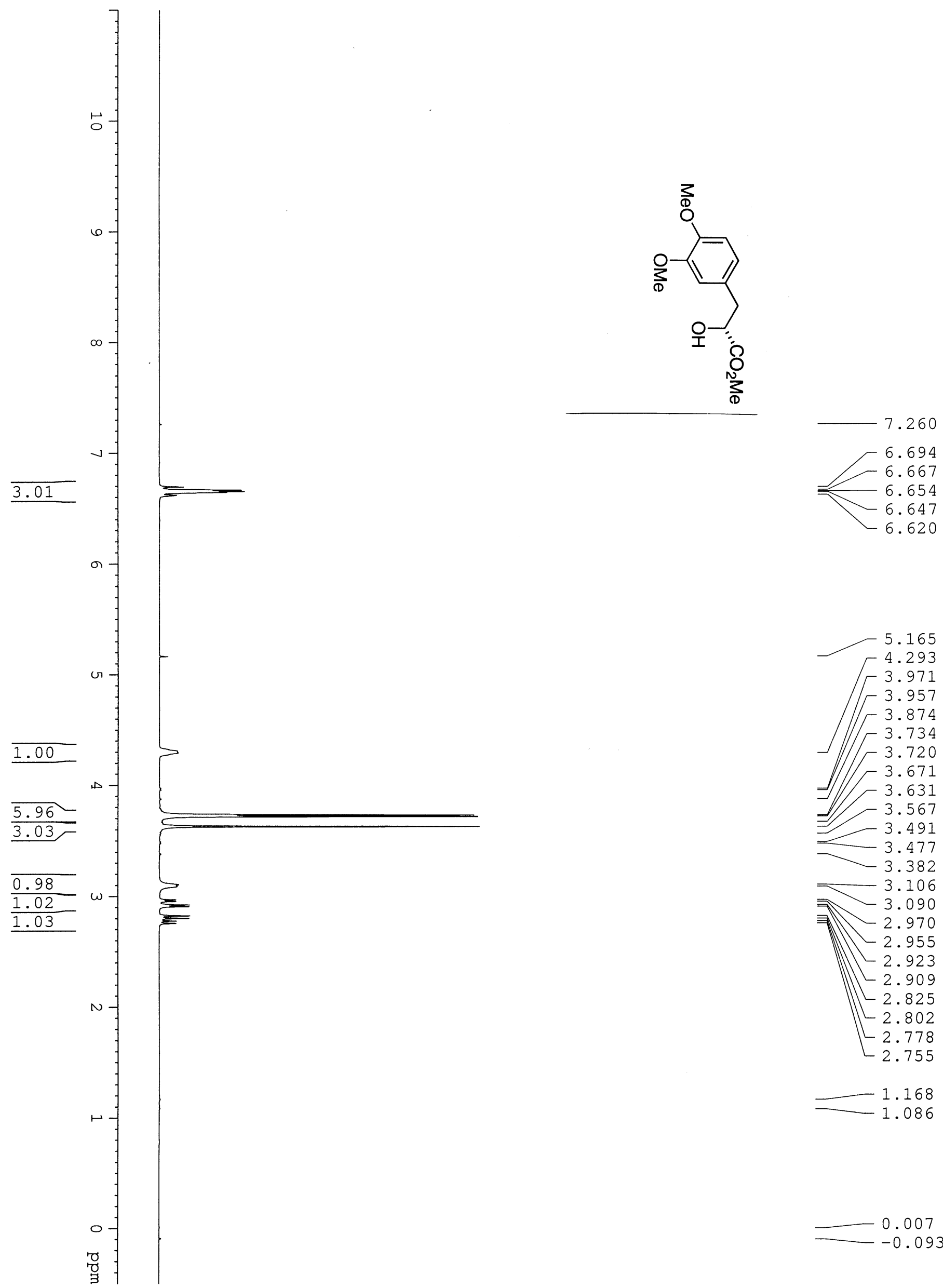


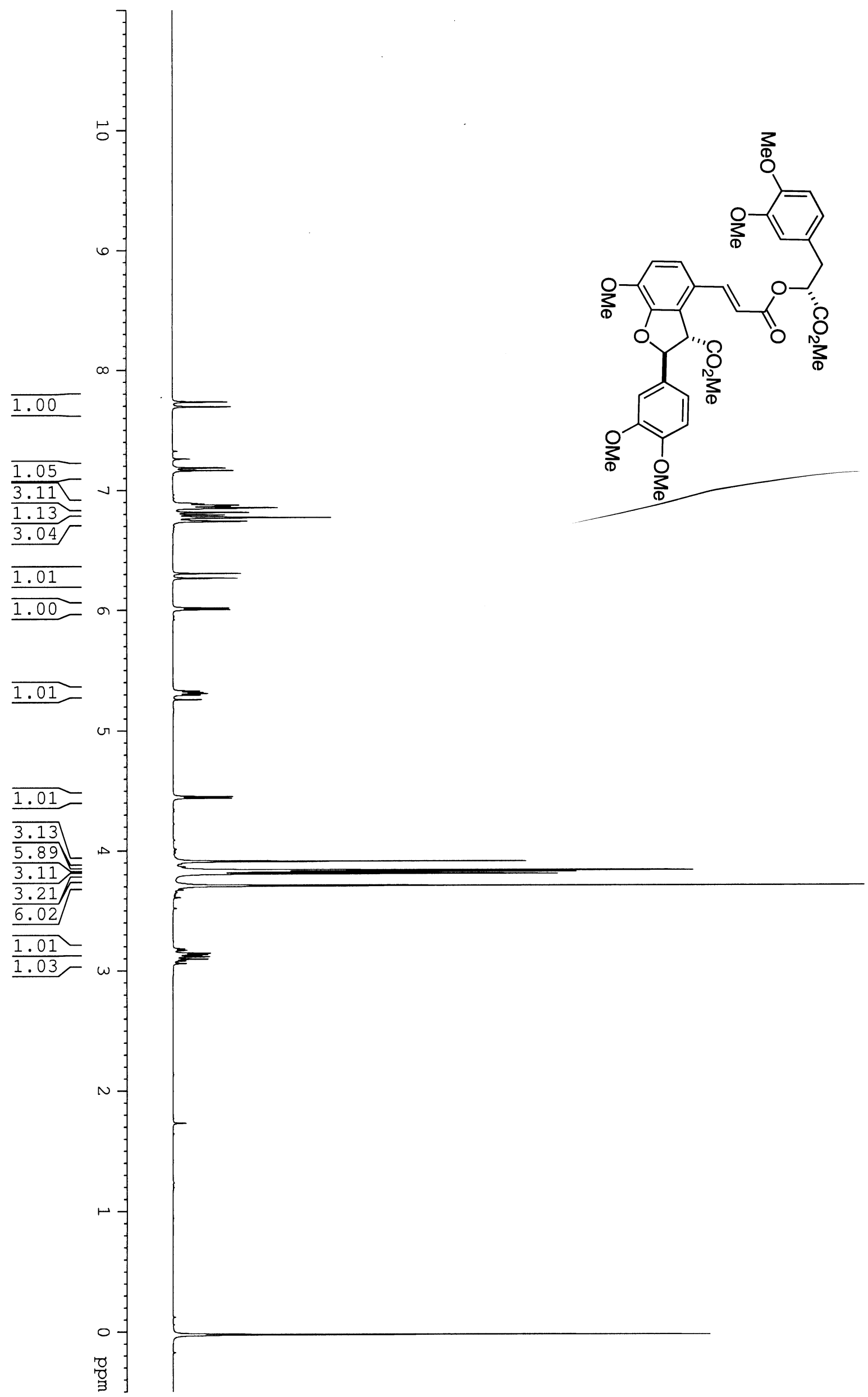



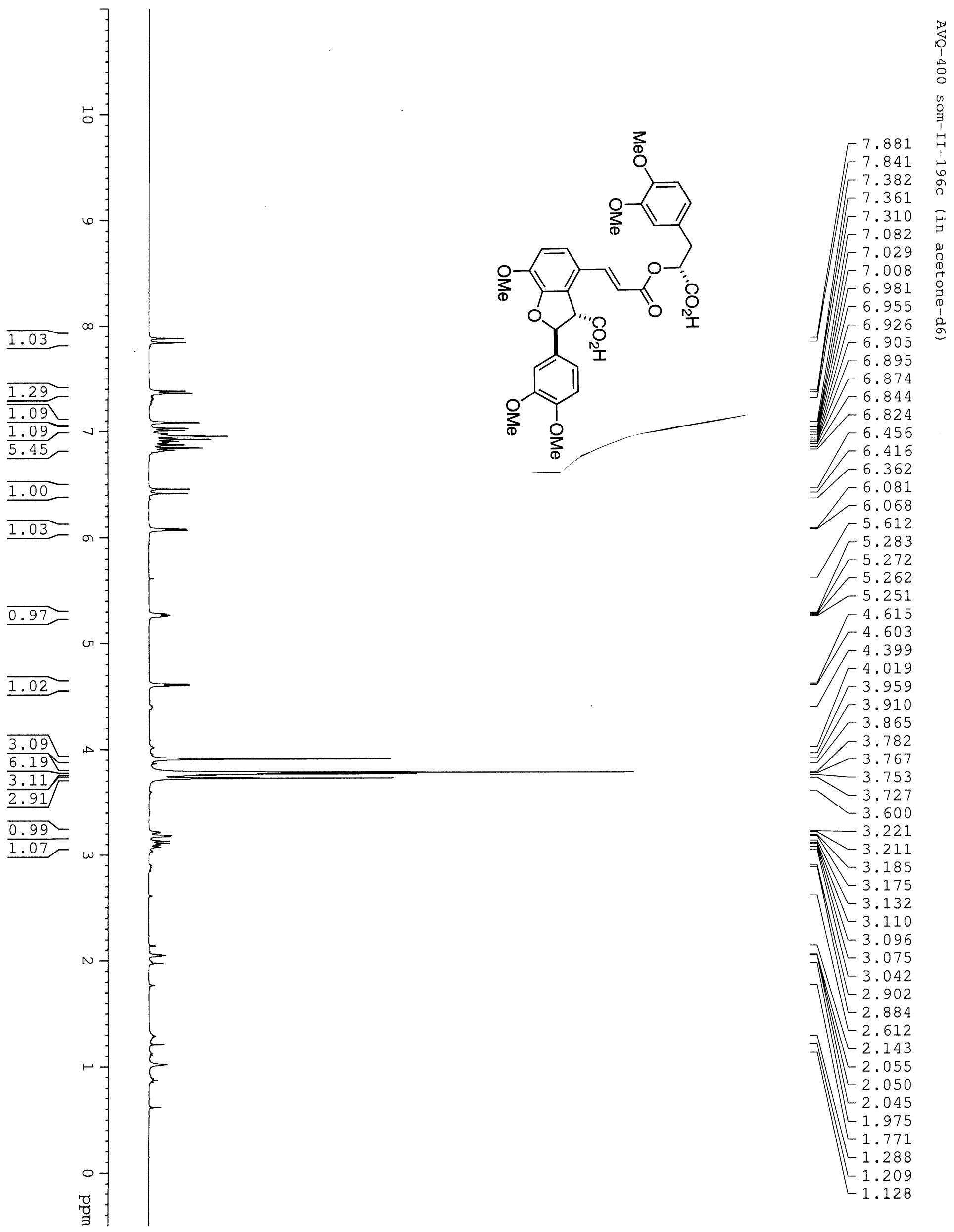


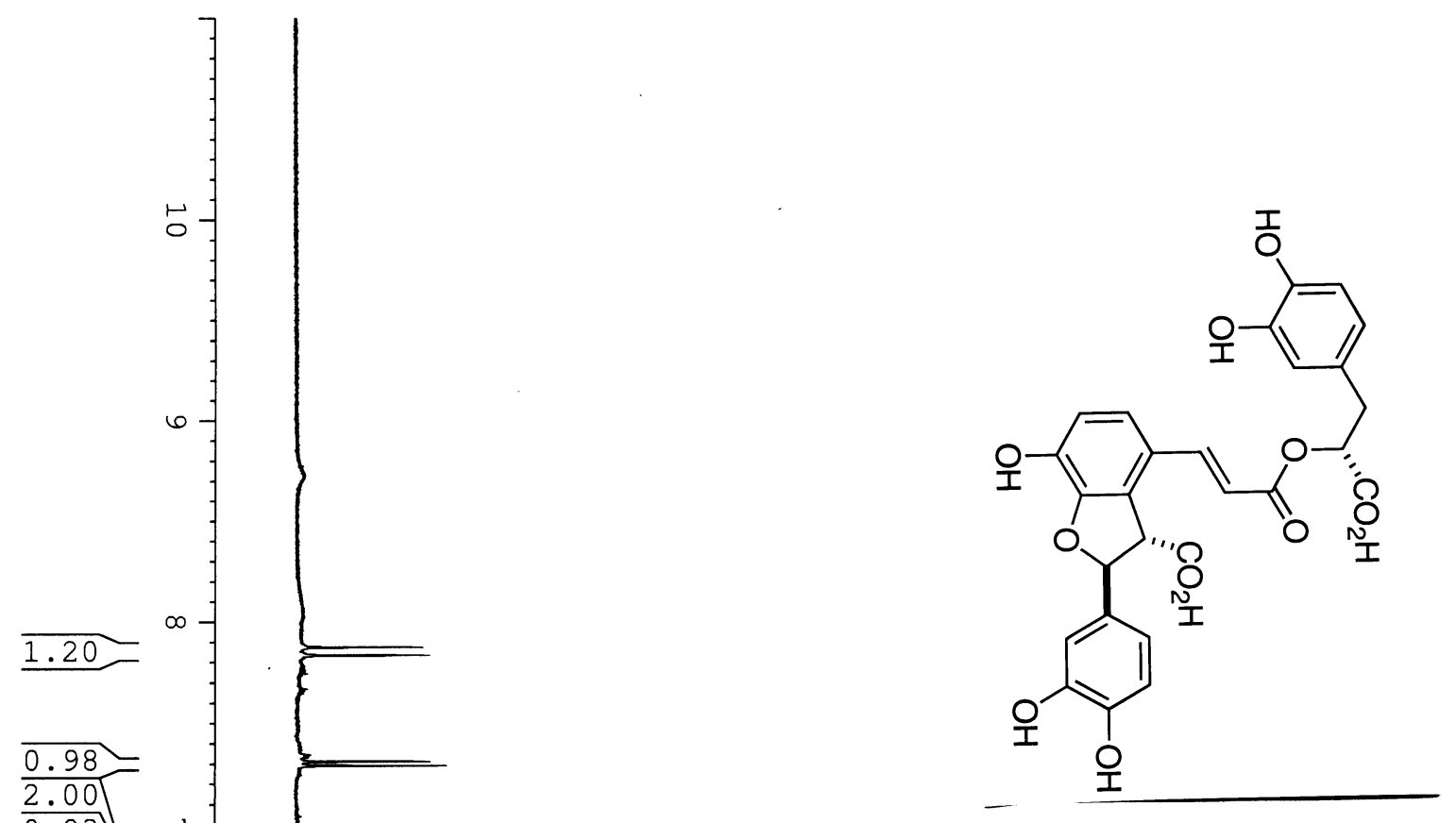

1
0
1
0
0
0

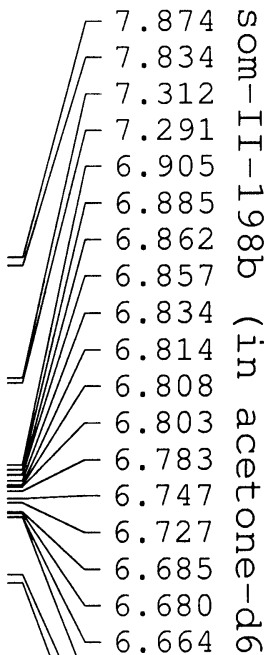

$7\left\lfloor\left[\begin{array}{l}6.664 \\ 6.660^{\circ}\end{array}\right.\right.$

L6.393

L6.353 C

-5.985 त

$\leftarrow 5.973$

$\longrightarrow-5.193$ \%

$-5.182$

$-5.171$

$-5.161$

$-4.533 \Omega$

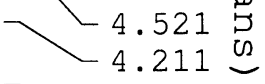

$=3.914$

$-3.816$

3.118

3.108

3.083

1.05

$\omega$

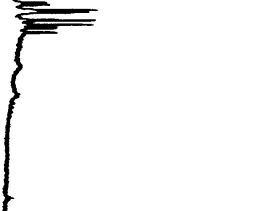

3.072

$-3.036$

$-3.014$

$-3.000$

2.979

2.086

N
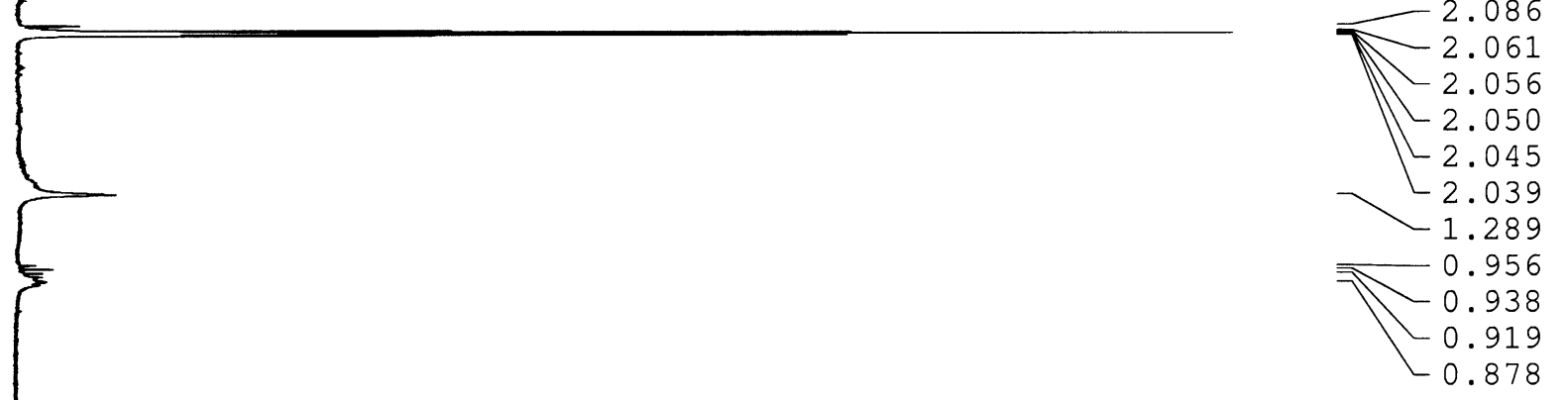

1
01
01
01

0.130 

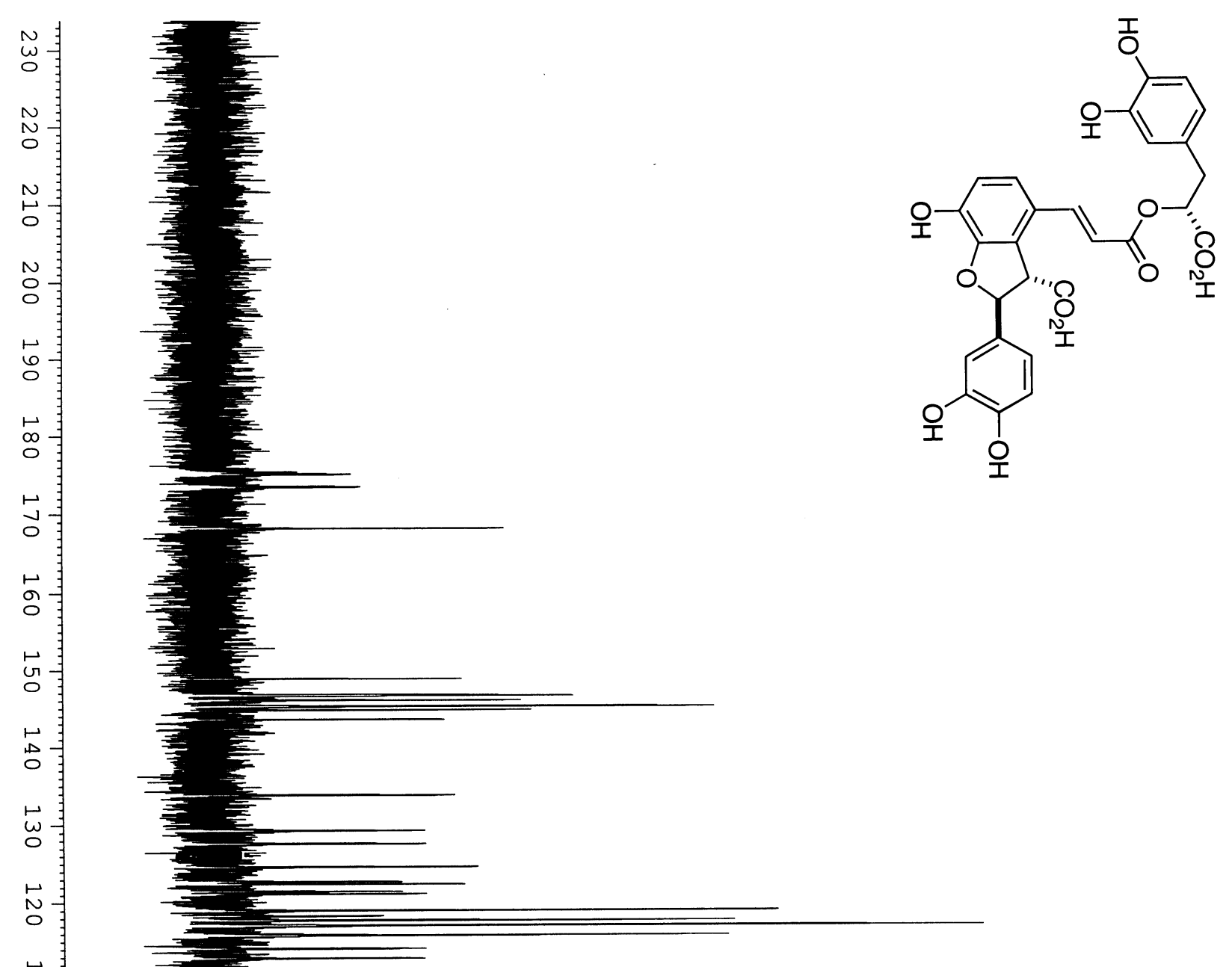

$\begin{array}{r}1124.61 \\ + \\ \hline\end{array} 122.39$

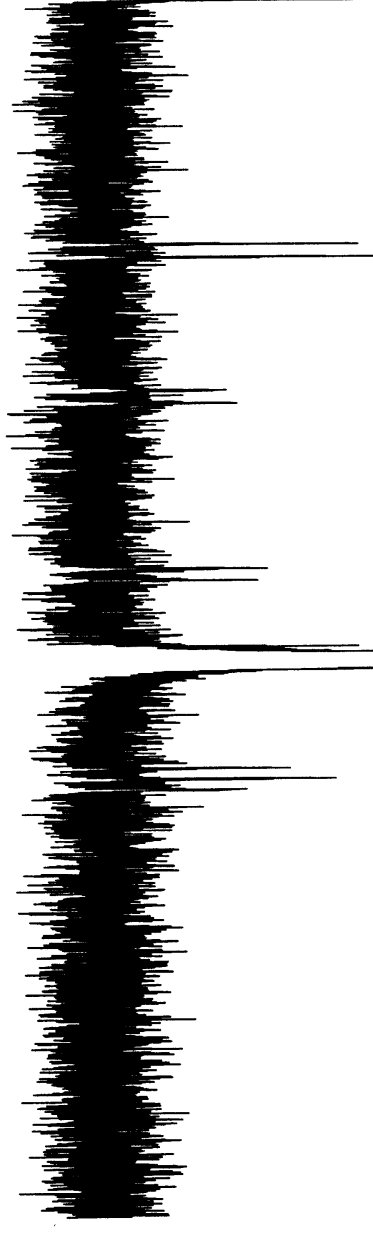

$-115.84$

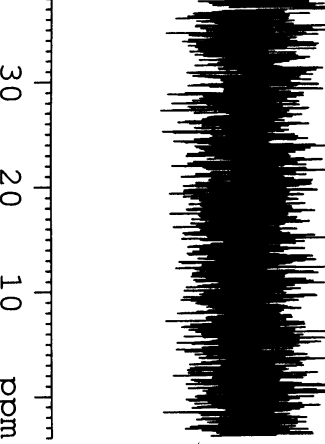

$-49.67$

$-49.50$

$-49.33$

49.16
-48.99

$-48.82$

48.65 
$: G N$
$:=$

: 3

$0 \Omega$
.$n$
.20

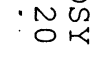

U 0

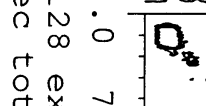

菓华.

$\stackrel{\rho}{P}$ : i

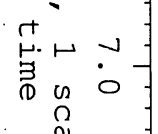

-

.

(D) ज $Q$

每

a

u

ir

u

$\triangleright$

ir

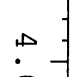

$\omega$

ir

$\omega$

o

$\therefore$

i

$N$

o

:

:

ir

0.0
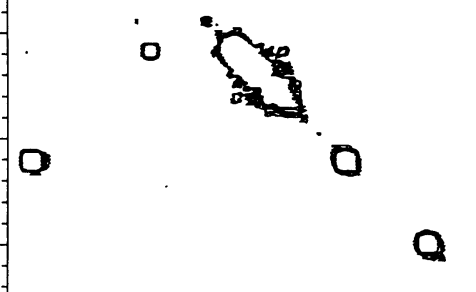

$a_{x}$

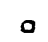

8

0
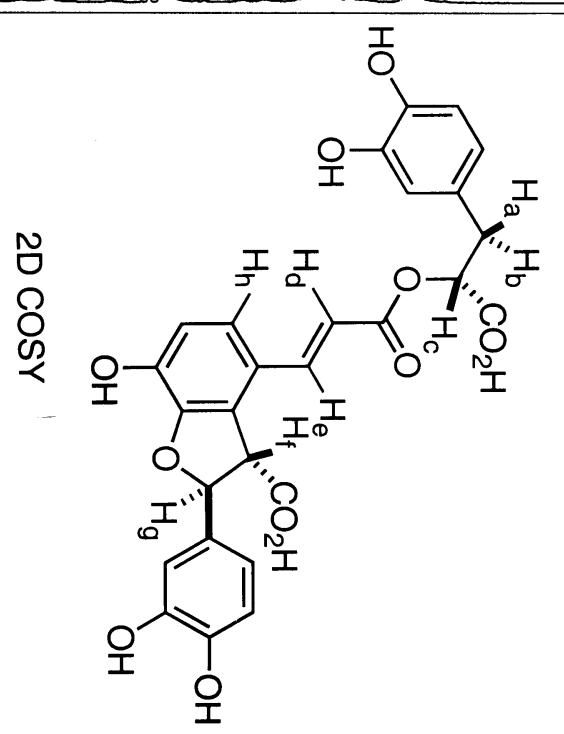

$\xi_{0}=$

$\infty$
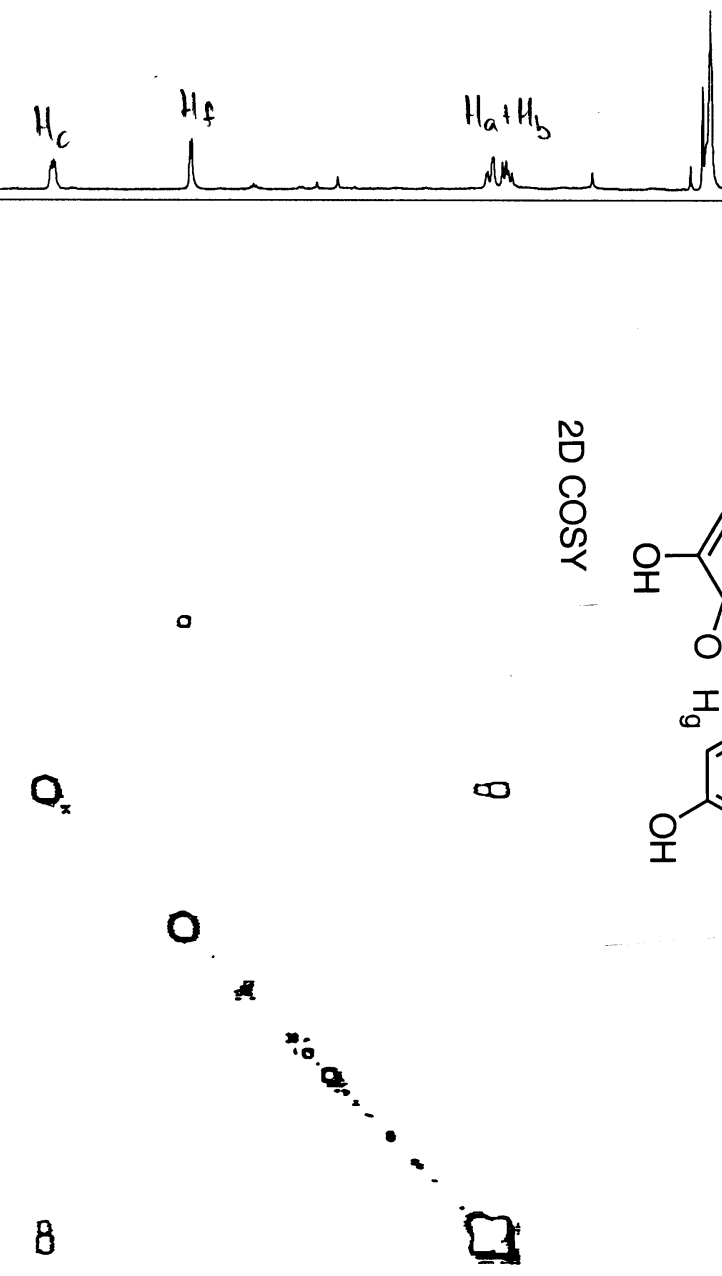

票 


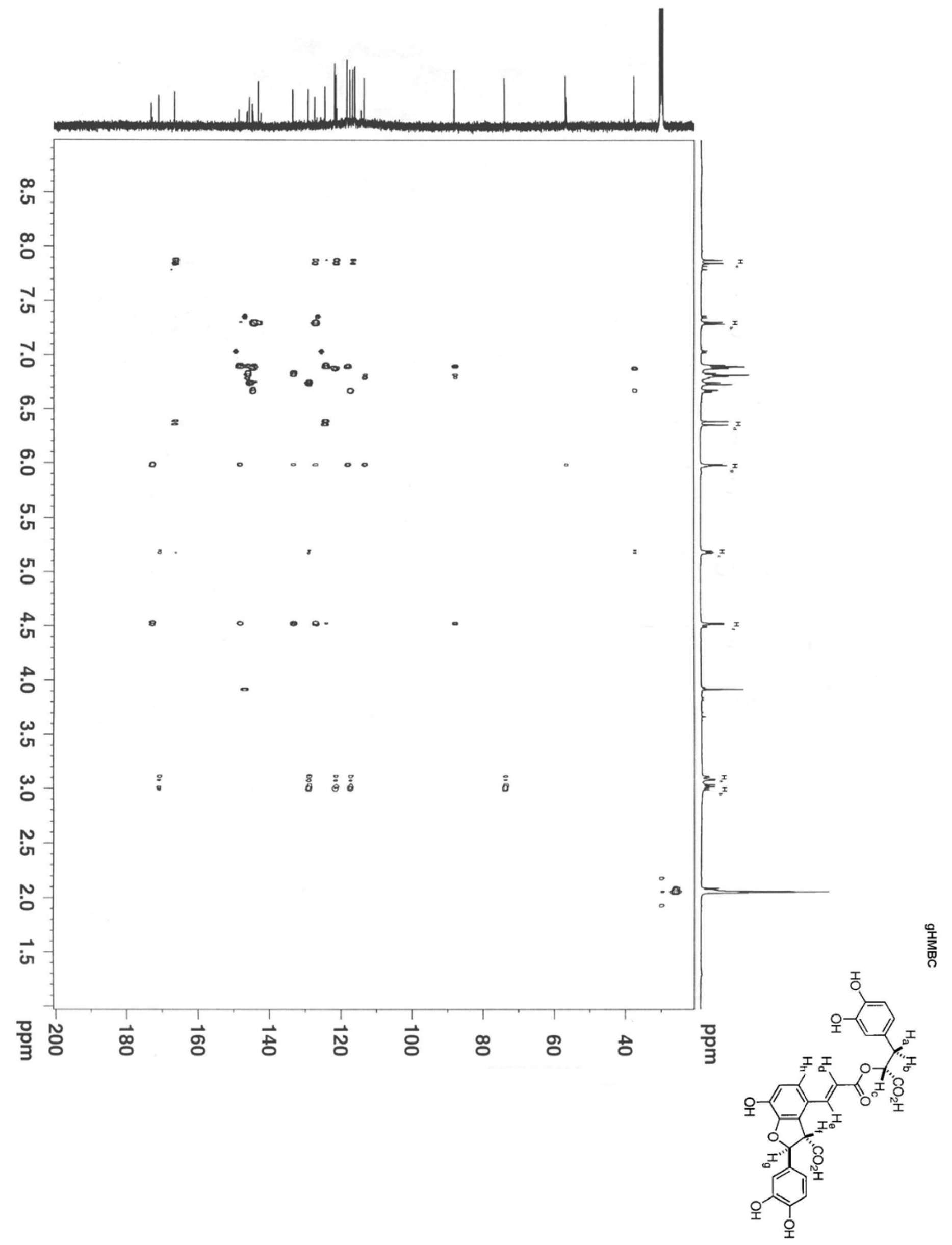



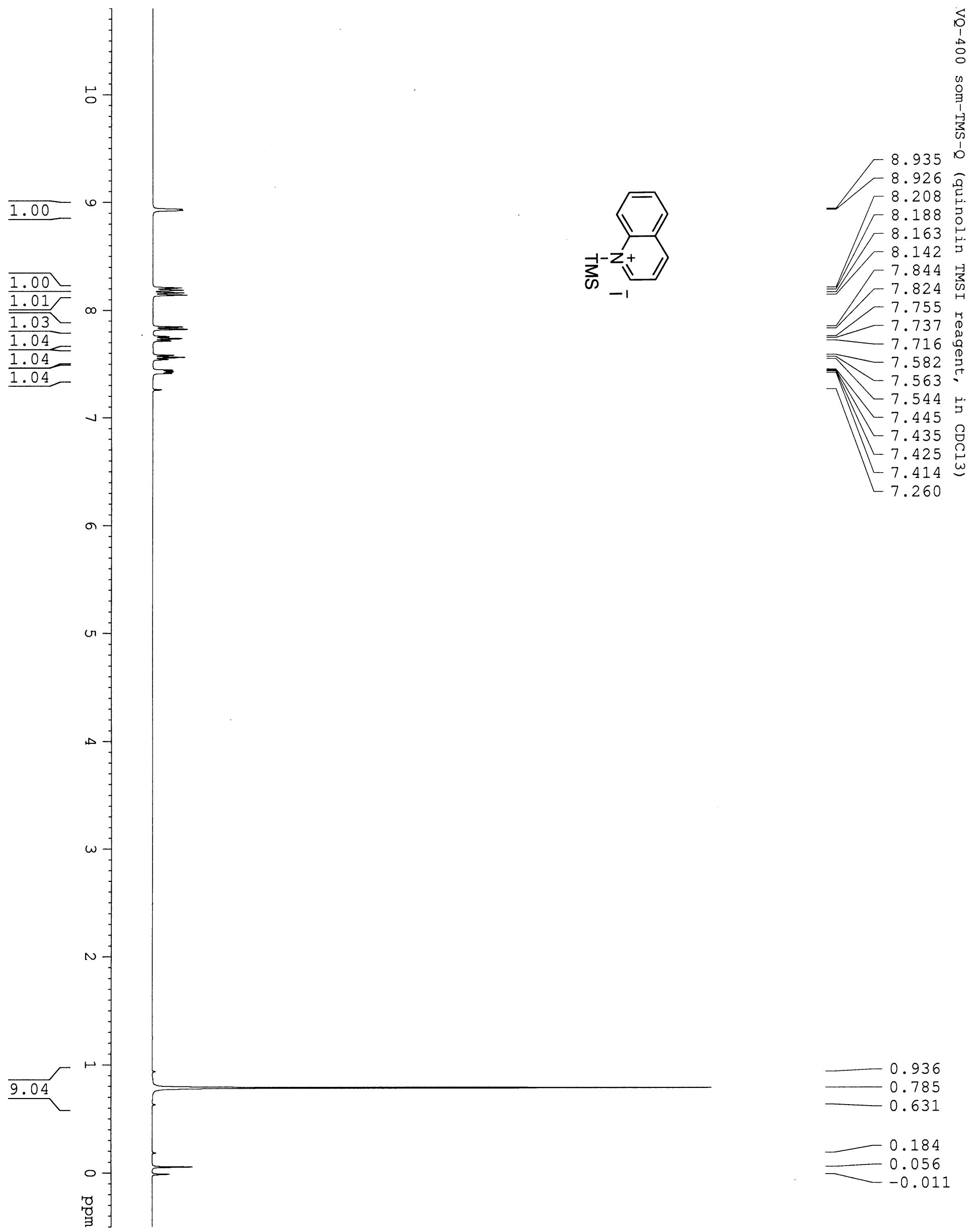

7.563 



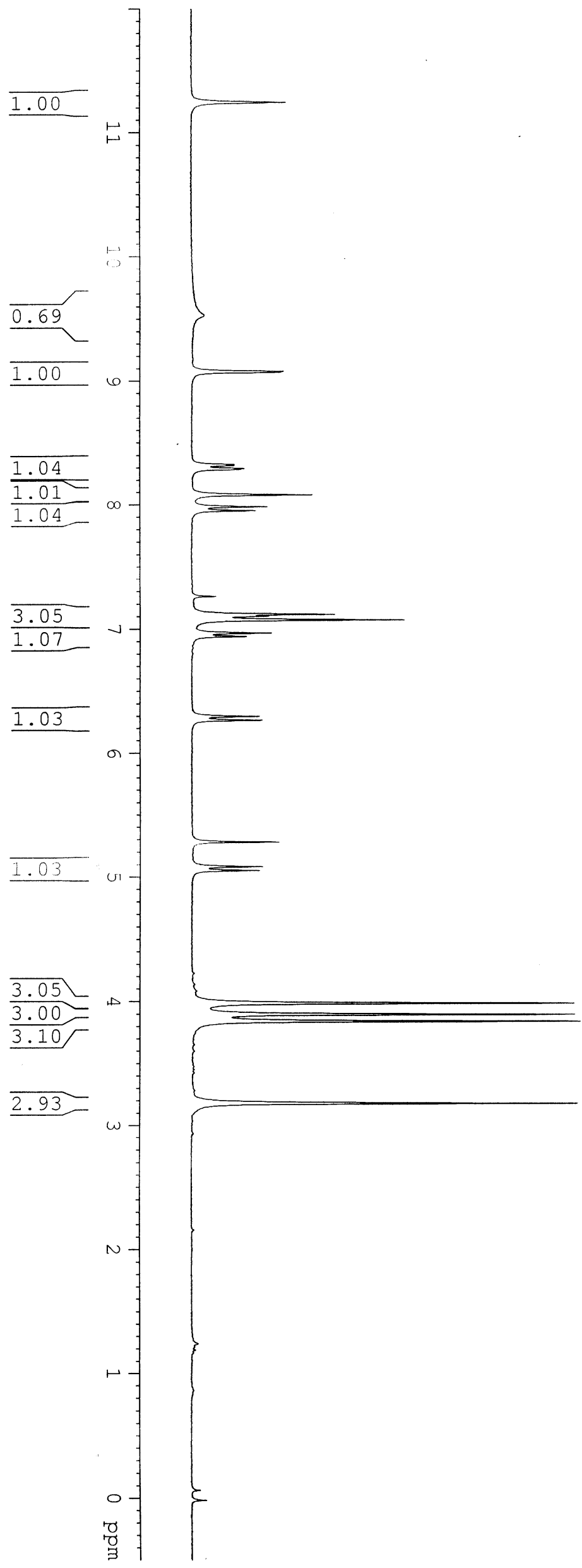

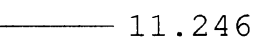
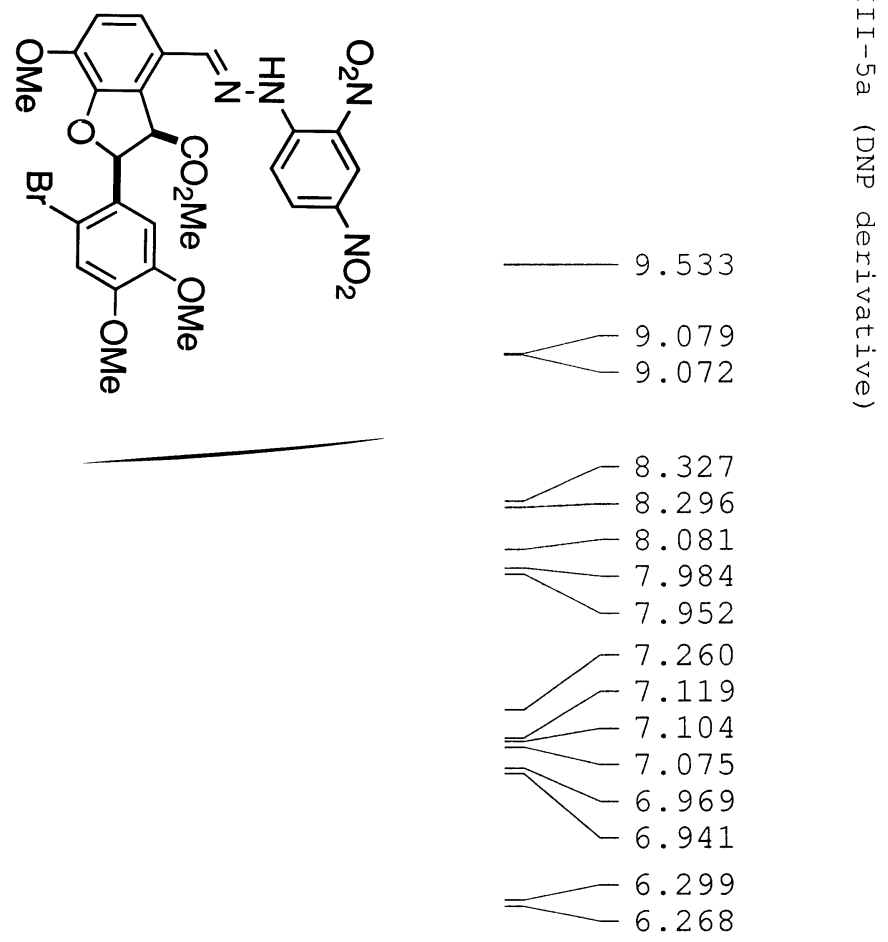

$-5.282$
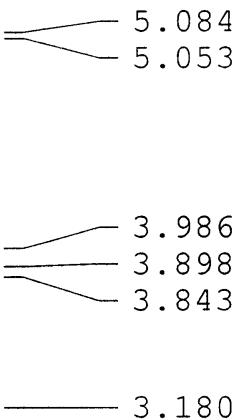

1. 240

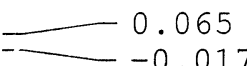

